\title{
Leaves and Fruits Preparations of Pistacia lentiscus L.: A Review on the Ethnopharmacological Uses and Implications in Inflammation and Infection
}

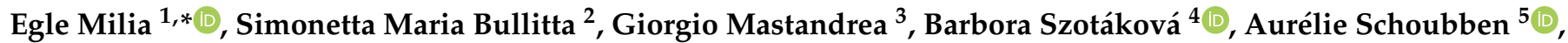 \\ Lenka Langhansová ${ }^{6}\left(\mathbb{0}\right.$, Marina Quartu ${ }^{7}$ (i), Antonella Bortone ${ }^{8}$ and Sigrun Eick ${ }^{9, *}$ (i)
}

Citation: Milia, E.; Bullitta, S.M.; Mastandrea, G.; Szotáková, B.; Schoubben, A.; Langhansová, L.; Quartu, M.; Bortone, A.; Eick, S. Leaves and Fruits Preparations of Pistacia lentiscus L.: A Review on the Ethnopharmacological Uses and Implications in Inflammation and Infection. Antibiotics 2021, 10, 425. https://doi.org/10.3390/ antibiotics 10040425

Academic Editor: William N. Setzer

Received: 19 February 2021

Accepted: 9 April 2021

Published: 12 April 2021

Publisher's Note: MDPI stays neutral with regard to jurisdictional claims in published maps and institutional affiliations.

Copyright: (c) 2021 by the authors. Licensee MDPI, Basel, Switzerland. This article is an open access article distributed under the terms and conditions of the Creative Commons Attribution (CC BY) license (https:// creativecommons.org/licenses/by/ $4.0 /)$.
1 Department of Medicine, Surgery and Experimental Sciences, University of Sassari, Viale San Pietro 43, 07100 Sassari, Italy

2 C.N.R., Institute for Animal Production System in Mediterranean Environment (ISPAAM), Traversa La Crucca 3, Località Baldinca, 07100 Sassari, Italy; simonettamaria.bullitta@cnr.it

3 Department of Biomedical Sciences, University of Sassari, Viale San Pietro 43/C, 07100 Sassari, Italy; 30050744@studenti.uniss.it

4 Faculty of Pharmacy, Charles University, Akademika Heyrovského 1203, 50005 Hradec Králové, Czech Republic; szotakova@faf.cuni.cz

5 Department of Pharmaceutical Sciences, University of Perugia, Via Fabretti, 48-06123 Perugia, Italy; aurelie.schoubben@unipg.it

6 Institute of Experimental Botany, Czech Academy of Sciences, Rozvojová 263, 16502 Prague, Czech Republic; langhansova@ueb.cas.cz

7 Department of Biomedical Sciences, University of Cagliari, Cittadella Universitaria di Monserrato, 09042 Cagliari, Italy; quartu@unica.it

8 Dental Unite, Azienda Ospedaliero-Universitaria di Sassari, 07100 Sassari, Italy; antonella.bortone@aousassari.it

9 Department of Periodontology, School of Dental Medicine, University of Bern, Freiburgstrasse 3, 3010 Bern, Switzerland

* Correspondence: emilia@uniss.it (E.M.); sigrun.eick@zmk.unibe.ch (S.E.); Tel.: +39-79-228437 (E.M.); +41-31-632-25-42 (S.E.)

Abstract: There is an increasing interest in revisiting plants for drug discovery, proving scientifically their role as remedies. The aim of this review was to give an overview of the ethnopharmacological uses of Pistacia lentiscus L. (PIL) leaves and fruits, expanding the search for the scientific discovery of their chemistry, anti-inflammatory, antioxidative and antimicrobial activities. PlL is a wild-growing shrub rich in terpenoids and polyphenols, the oil and extracts of which have been widely used against inflammation and infections, and as wound healing agents. The more recurrent components in PIL essential oil (EO) are represented by $\alpha$-pinene, terpinene, caryophyllene, limonene and myrcene, with high variability in concentration depending on the Mediterranean country. The antiinflammatory activity of the oil mainly occurs due to the inhibition of pro-inflammatory cytokines and the arachidonic acid cascade. Interestingly, the capacity against COX-2 and LOX indicates PIL EO as a dual inhibitory compound. The high content of polyphenols enriching the extracts provide explanations for the known biological properties of the plant. The protective effect against reactive oxygen species is of wide interest. In particular, their anthocyanins content greatly clarifies their antioxidative capacity. Further, the antimicrobial activity of PIL oil and extracts includes the inhibition of Staphylococcus aureus, Escherichia coli, periodontal bacteria and Candida spp. In conclusion, the relevant scientific properties indicate PIL as a nutraceutical and also as a therapeutic agent against a wide range of diseases based on inflammation and infections.

Keywords: essential oils; terpenoids; polyphenols; water extracts; ethanol extracts; natural antimicrobials; natural anti-inflammatory; Mediterranean plants; pharmaceutical plants 


\section{Introduction}

The undesirable side effects of antibiotics in addition to increasing microbial resistance have created a demand for new alternative molecules [1]. Non-steroidal anti-inflammatory drugs (NSAIDs) or even steroids, often inducing toxic side-effects [2] have also gained further interest as molecules presenting an anti-inflammatory character.

On these bases, there is an increasing attention towards revisiting plants for drug discovery, proving scientifically their role as popular remedies to diseases [3,4]. Approved therapeutic agents such as statins, tubulin-binding anticancer drugs and some types of immunosuppressants are examples of molecules originating from natural plants [5].

In the Mediterranean region, folk medicine has used extracts and oils derived, e.g., from Malva species [1], Thymbra capitate (L.) Cav. [2] and Olea europea L. [3] for many years.

The biological activity of essential oils (EOs) and polyphenols from plants and herbs is related to the presence of different chemical classes. In this regard, terpenes and terpenoids in EOs are promising agents in the prevention and treatment of diseases [6,7]. Terpenes are hydrocarbons, and terpenes containing additional functional groups, usually oxygen-containing, are called terpenoids [8]. They are lipophilic, and interact with cell membranes, neuronal and muscle ion channels, neurotransmitter receptors, G-protein coupled receptors, second messenger systems and enzymes [9]. Their beneficial effects and their roles have been evaluated for many decades in human disease, such as inflammatory diseases, tumorigenesis and neurodegeneration using cell and animal models, suggesting terpenes and terpenoids as potential chemopreventive and therapeutic agents [10]. Further interesting capabilities have been ascribed to polyphenols from botanical species, with molecules including tannins, flavonoids and lignin-carbohydrate complexes having been associated with strong antimicrobial, anti-inflammatory and antioxidant properties [11].

In this context, Pistacia lentiscus L. (PIL) is a wild-growing shrub rich in terpenoids and polyphenols [12]. PIL includes numerous wild and cultivated species, distributed in the Mediterranean and Middle Eastern areas. Although there has been broad investigation on the aromatic natural resin and its clinical application $[13,14]$, the scientific data regarding PIL oil or extracts of leaves, fruits and woods have been not summarized yet. Today, the scientific interest in these edible and not-edible parts of PIL is wide-spreading, as some studies underlined the potential benefit against inflammation and infections $[12,15,16]$. Additionally, the high content of polyphenols found in the extracts make them attractive against chronic and degenerative diseases and as nutraceuticals in human health [15].

Given the above considerations, the purpose of this review was to screen the biological properties of PIL EO and extracts of leaves and fruits. Starting from this, we searched for the phytochemistry of PlL growing in the different geographical areas and reported on the anti-inflammatory and antimicrobial abilities of the plant. For that purpose, a search for the existing literature was made by using data bases such as MEDLINE/PubMed and the Cochrane Library electronic databases.

\section{Botany and Taxonomy}

\section{PlL Belongs to the Genus Pistacia, Anacardiaceae Family, Order Sapindales}

Different classifications have been proposed regarding the Pistacia genus. One of the most known is that of Zohary [16], who classified the genus into four main groups according to the characteristics of the leaf and nut morphology (Table 1).

In the Mediterranean area (Middle East and Europe), the three most represented species are the following:

1. Pistacia vera L., which is characteristic of the temperate areas of Asia Minor, and grown abundantly in Greece, the Aegean Islands and in Sicily (Italy). This species has been known since ancient times as attested in reports of the Old Testament. Additionally, there have been notices of Pistacia vera by Persian and Greek populations since the 6th and the 3rd century B.C., respectively.

2. Pistacia terebinthus L., originating from the island of Chios, has spread to all the Mediterranean coasts over the centuries. Today, it is mostly present in Portugal, 
Palestine and North Africa, and in the Middle East of Asia till the western borders of India. In Italy, it is mainly found in the southern part of the peninsula and in Sardinia and Sicily.

3. Pistacia lentiscus L., also known as mastic tree or lentisk (Figure 1).

Table 1. Taxonomy description (groups and species) of the Pistacia genus (adapted from Zohary [16]).

\begin{tabular}{cc}
\hline Group & Species \\
\hline Lenticella & Pistacia mexicana HBK \\
\hline Eu lentiscus & Pistacia texana Swingle \\
\hline & Pistacia lentiscus L. (mastic tree) \\
\hline Butmela & Pistacia saporte Burbar \\
\hline Eu terebintus & Pistacia weinmannifolia Poisson \\
\hline & Pistacia atlantica Desf. \\
\hline & Pistacia chinensis Bge. \\
\hline & Pistacia khinjuk Stocks \\
\hline & Pistacia palaestina Bois. \\
\hline & Pistacia terebinthus L. \\
\hline
\end{tabular}

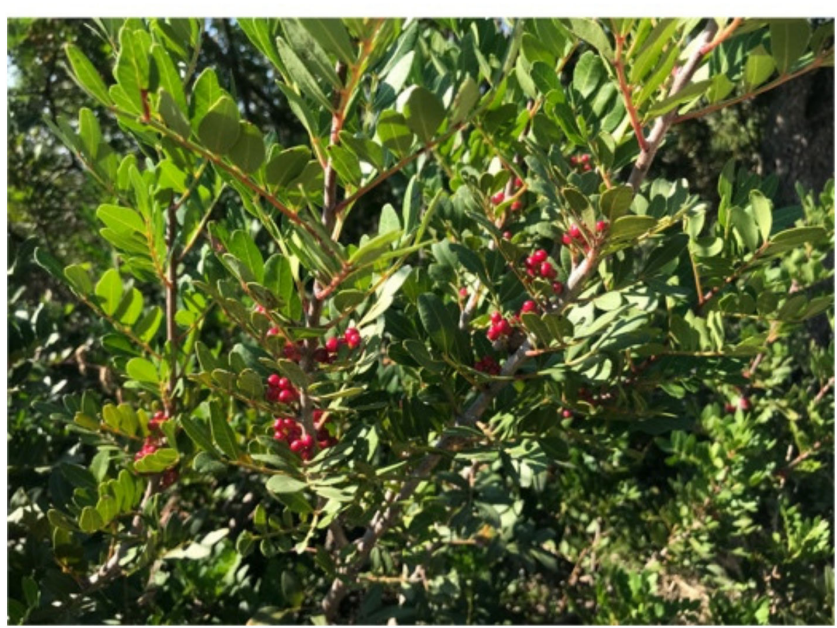

Figure 1. Pistacia lentiscus L., also known as mastic tree or lentisk.

PlL represents one of the most typical shrubs in the Mediterranean maquis (shrubland) of Europe, Morocco, Turkey, Iraq and Iran [17]. In Italy, it is characteristic of the sensitive ecosystem, like that of Sardinia [18], where it grows along the coast up to $700 \mathrm{~m}$ above sea level.

PlL is an evergreen environmentally sustainable shrub. It is well-adapted to harsh growing conditions, dryness and a warm environment, which all exercise an influence on the genotype and richness of secondary metabolites [19]. The plant is dioecious, where male and female flowers are on independent trees. The leaves are leathery, bright green and alternate. They are arranged in compound, pinnate whorls. The unisexual flowers are grouped in clusters. The globular fruit is a fleshy drupe, which ripens in August and ranges in color from red to brown in view of the different degrees of maturity [17]. PlL can develop leaf galls due to insect attack, particularly aphid attacks [20]. Common aphid species, such as Slavum wertheimae and Baizongia pistaciae L., manipulate the leaves to form tumorous galls for the safety and nutriment of their larvae [21]. The galls are rich in volatile-like terpenes with an abundance of monoterpenes, $\alpha$-pinene and limonene [22]. Their chemical 
composition differs from that of the healthy leaves, which have in general a higher content of sesquiterpenes [22].

\section{Historical and Cultural Use}

PIL has had a wide range of applications over the centuries. One of the oldest dates back to the Nuragic civilization (1800 to 238 BCE) and was ascribed to the Sardinian population: the oil obtained by cold-pressing the berries was widely used for social purposes, i.e., home or votive lighting lamps, cooking, as well as as a popular remedy [17] This habit is attested to by the presence of residues of "olium lentiscinum" often found during archaeological excavations in "torcularia" (ancient oil mills) [18].

Today, PIL is considered as an environment phytostabilizer due to the ability to detoxify the soil from harmful pollutants and heavy metals [23]. Furthermore, the plant represents an important source to increase milk quality and dairy products from ruminants browsing the Mediterranean maquis [24].

\section{Ethnopharmacology}

The ethnopharmacological survey on the medicinal use of PIL is reported in Table 2.

Table 2. Ethnopharmacological uses of Pistacia lentiscus L.

\begin{tabular}{|c|c|c|}
\hline Geographical Area & Ailment/Uses & Ref. \\
\hline Sardinia, Italy & $\begin{array}{l}\text { Oral cavity inflammation and infection, tooth ache, osteoarthritis, } \\
\text { bronchitis, cough sedative, antipyretic, allergies, asthma, ulcerations, } \\
\text { gastrointestinal disorders, wound healing and haemostatic }\end{array}$ & {$[19,25-31]$} \\
\hline $\begin{array}{l}\text { Southern regions of Italy } \\
\text { (Calabria and Campania) }\end{array}$ & $\begin{array}{l}\text { Inflammation of the mouth, tooth ache, mycosis, herpes and refreshing } \\
\text { feet }\end{array}$ & {$[27,32]$} \\
\hline $\begin{array}{l}\text { Central regions of Italy (Abruzzi, } \\
\text { Marche and Tuscany), Spain }\end{array}$ & Hypertension and cardiac diseases & {$[27,33]$} \\
\hline Spain & Analgesic, teeth strengthening, hypertension and cardiac diseases & {$[33,34]$} \\
\hline Tunisia & $\begin{array}{l}\text { Antipyretic, astringent, eczema, paralysis, antimicrobial, throat } \\
\text { infections, asthma, hypertension, cardiac diseases, paralysis, diuretic } \\
\text { properties, renal stones, jaundice, antiatherogenic effect, } \\
\text { antihepatotoxic and gastrointestinal diseases }\end{array}$ & {$[27,35]$} \\
\hline Algeria & Stomach ache, dyspepsia, peptic ulcer, diarrhea and rheumatism & [36] \\
\hline $\begin{array}{l}\text { Morocco and } \\
\text { North Africa }\end{array}$ & Hypertension, cardiac diseases and diabetes & [37] \\
\hline Libya & Immuno-stimulant and antimicrobial & [23] \\
\hline Jordan & Ameliorate jaundice & {$[38,39]$} \\
\hline Israel & Heartburn and soothes stomach & [40] \\
\hline Iran & $\begin{array}{c}\text { Gum tissue strengthened, breath deodorizer, brain and liver tonic and } \\
\text { gastrointestinal ailments }\end{array}$ & {$[41,42]$} \\
\hline Turkey & $\begin{array}{l}\text { Throat infections, asthma, eczema, stomach ache, renal stones, } \\
\text { paralysis, diarrhea, jaundice, anti-inflammatory, antimicrobial, } \\
\text { antipyretic, stimulant and astringent }\end{array}$ & [43] \\
\hline
\end{tabular}

As previously referred to, there are few written indications on the medicinal use of the oil as a crude compound, or of the extracts as well. Drinking water extracts and topical application of the extracts or even whole parts of the plant (woods or leaves) have been the most common means of antagonizing gastrointestinal, hepatic, urinary, pulmonary and neurological diseases. In fact, the medicinal value of PlL in popular medicine covers a wide range of diseases, mainly including inflammatory processes and infections.

The Sardinian population has always found the medicinal properties of PIL highly appealing. A large number of publications report PIL oil and water extracts as useful 
means against a wide variety of inflammatory diseases, infections, allergies [21,27,28,32,33] and gastrointestinal disorders [28-30], and as wound healing [31]. It is further interesting to note that the Sardinian population administrated PIL as a smoke obtained by burning or boiling the soft wood and leaves, particularly in the cases of osteoarthritis, bronchitis and allergies [30]. In addition, PIL is still used as a remedy toward tooth ache and gingival inflammation by administering extracts from the leaves as oral mouthwash, beverages or by directly chewing the soft stems and leaves [30].

Similar beneficial effects have been reported by using the plants growing in the Southern (Campania) [32] and in the center (Abruzzo, Marche and Toscana) of Italy, in Tunisia [27] and in Spain [34].

PIL has been one of most used plants in Israeli and neighboring countries' traditional pharmacology [40]. In Jordan, it was commonly used to antagonize jaundice [38,39]. In Algeria, it is known as antimicrobial, antioxidant, hypotensive and hypoglycemic agent [44]. In Morocco and Tunisia [35,37], PlL has been largely used as a remedy against gastrointestinal, kidney and hepatic disorders, in addition to being used to treat hypertension, diabetes, cardiac diseases, coughs, sore throats and eczema. Similar applications are reported in Turkey [43] and in Iran [41,42]. Meanwhile, in Tunisia, Spain and in the center of Italy, it has emerged as an agent against hypertension and heart diseases [27,37,41-43].

Regarding the veterinary use, in Sardinia, domestic animals are still treated by PlL wood to combat gastrointestinal disorders, and by swab bark in wound healing procedures and skin diseases [45]. Meanwhile, in Spain, the leaves are mentioned to treat specifically canine distemper [33].

\section{Phytochemical Constituents}

Mainly leaves and fruits are used for preparations of EO and also of water and alcoholic extracts. In Table 3, the major compounds of the EO and the extracts are listed. Hydro-distillation using Clevenger-type devices, and ethanol solvent have been the more common methods to obtain, respectively, the oil and extracts from leaves and fruits. However, the oil obtained by hydro-distillation and the extracts by solvents can have different organoleptic profiles and chemical compositions. These differences, in turn, will affect some properties, among which is the antimicrobial capacity, which is reported to be higher in a material solvent extract in comparison to a hydro-distilled [46]. Additionally, gas chromatography-mass spectroscopy (GC/MS) and high-performance liquid chromatography (HPLC) have been the most useful means to quantify phytochemically the oils and extracts, respectively [47].

PIL EO is constituted by a mixture of terpenes and terpenoids, mainly monoterpenes and sesquiterpenes, which are also responsible for the characteristic smell and flavoring of the plant $[12,16,21,31]$. It has been reported that terpenes in PIL are more genetically than environmentally related [48]. Nevertheless, the environment of growing, seasonability of harvesting and kind of material (edible or not-edible parts of the plant) have to be considered when explaining the differences in chemistry of the oils and extracts [19]. Up to 64 chemical constituents have been reported in the PIL EO fingerprint, in addition to other fractions that cannot be quantified by the assays [49]. Some of these terpenoids are constituent fractions of cannabis sativa [9], and called "non-cannabinoids terpenoids". In PIL oil, non-cannabinoid terpenoids are more likely to be represented by $\alpha$-pinene, myrcene, limonene, (E)- $\beta$-caryophyllene and $\gamma$-terpinene (Table 3 ). They are also included in the list of "terpene super classes" [9]. Furthermore, it is appealing to report that when non-cannabinoid terpenoids reach a concentration equal to or higher than $0.05 \%$ in an oil, they can confer pharmacological properties to such an oil, which can be classified as pharmaceutically active [50].

In view of the prevalent fractions of monoterpenes and oxygenated sesquiterpenes, the EO can be grouped into different chemotypes [17]. In this regard, the recurrent higher amount of $\alpha$-pinene (16.9-19.5\%) and terpinen-4-ol (7.7-16.5\%) in comparison to the other compounds, allowed the classification of the oil from the leaves of PlL growing in Sar- 
dinia as the $\alpha$-pinene/terpinen-4-ol chemotype [19,49]. Similarly, the Greek oil from PIL leaves is the $\alpha$-pinene/terpinen-4-ol chemotype [51,51]. Nevertheless, the simultaneous existence of different chemotypes in a place can be justified by dissimilar geographical sites of harvesting in that country. An example is represented by the Corsican chemotype, which is expressed by three main phenotypes: the first is $\alpha$-pinene/terpinen-4-ol; the second is terpinen-4-ol/limonene; and the third is myrcene-rich (88\%) [19]. Very characteristic is the high content of $\delta$-3-carene (65\%) in the Egyptian oil [52], while the monoterpene terpinen-4-ol, together with $\alpha$-pinene and the sesquiterpene myrcene, are among the higher represented fractions in the chemistry of PIL EO from Spain, Morocco and Turkey [53-55]. Conversely, $\alpha$-pinene $(65-86 \%)$ and $\beta$-myrcene $(3 \%)$ are the major fractions which characterize the oils of mastic from plants growing in Spain [53].

Regarding the sesquiterpenes, limonene, $\alpha$ - and $\beta$-caryophyllene, D-germacrene, $\delta$ cadinene and $\alpha$-cadinol, $\beta$-bisabolene, $\beta$-bourbonene and caryophyllene oxide, they have shown extremely variable concentrations in PIL EO $[16,21,53,56]$. With respect to PIL leaves oil, the fruits oil changes significantly in its chemistry: limonene, sabinene and myrcene have been identified as the main representative fractions, in addition to $\alpha$-pinene [56]. Additionally, the oil from the berries is rich in anthocyanins [57], which in addition to the concentration of fatty acids, such as oleic acid and linoleic acid [58], are precious as antioxidant compounds [59].

Furthermore, comprehensive studies have been conducted analyzing methanol and alcohol extracts of PIL leaves, while at the same time investigating their chemical profile, where a high concentration of phenolproponoids is reported (Table 3 ).

In this regard, an interesting study was carried out by Romani and co-workers [60]. Using ethyl acetate and methanol fractions of PlL leaves, the authors identified a high polyphenol content in the extracts, which represented $7.5 \%$ of the leaf dry-weight. In this content, three major classes of secondary metabolites were identified: (i) gallic acid and galloyl derivatives of both glucose and quinic acid; (ii) flavonol glycosides, i.e., myricetin and quercetin glycosides; and (iii) anthocyanins, namely delphinidin 3-O-glucoside and cyanidin 3-O-glucoside. All of these represent strong antioxidant polyphenols, which have implications in the prevention of chronic and inflammatory diseases [59]. Additionally, 46 different compounds were identified in the methanol extracts of PIL leaves from plants growing in Algeria [61].

Table 3. Chemical profiles of Pistacia lentiscus L.

\begin{tabular}{|c|c|c|c|c|}
\hline Plant Material & Origin & Main Components of Essential Oils or Plant Extracts & Test Assays & Ref. \\
\hline \multicolumn{5}{|c|}{ Essential oils from } \\
\hline Leaves & Spain & $\begin{array}{c}\beta \text {-myrcene }(19 \%), \alpha \text {-terpineol }+ \text { terpinen-4-ol }(15 \%), \alpha \text {-pinene } \\
(11 \%)\end{array}$ & GC-MS & [53] \\
\hline $\begin{array}{l}\text { Unripe fruit } \\
\text { Ripe fruit }\end{array}$ & & $\begin{array}{c}\beta \text {-myrcene }(54 \%), \alpha \text {-pinene }(22 \%) \\
\beta \text {-myrcene }(19 \%), \alpha \text {-pinene }(11 \%), \delta \text {-3-carene }\end{array}$ & $\begin{array}{l}\text { GC-MS } \\
\text { GC-MS }\end{array}$ & \\
\hline Leaves & Egypt & $\delta$-3-carene $(65 \%)$, sesquiterpene alcohols $(4 \%)$ & GC-MS & [52] \\
\hline Leaves & Greece & $\begin{array}{l}\text { Myrcene }(20.6 \%) \text {, germacrene D (13.3\%), E-caryophyllene } \\
(8.3 \%), \alpha \text {-cadinol }(7.3 \%) \text {, t5-cadinene }(7.0 \%)\end{array}$ & GC-MS & [62] \\
\hline Leaves & Turkey & $\begin{array}{c}\text { Terpinen-4-ol }(29.9 \%), \alpha \text {-terpineol, }(11.6 \%) \text {, limonene }(10.6 \%), \\
\text { (Z)-3-Hex-1-enyl benzoate }(6.7 \%), \alpha \text {-pinene }(4.2 \%), \\
\beta \text {-caryophyllene }(3.2 \%)\end{array}$ & GC-MS & [63] \\
\hline Leaves & Morocco & $\begin{array}{l}\text { Myrcene }(39.2 \%), \text { limonene }(10.3 \%), \beta \text {-gurjunene }(7.8), \\
\text { germacrene }(4.3 \%), \alpha \text {-pinene }(2.9 \%) \text {, muurolene }(2.9 \%)\end{array}$ & GC-FID; GC-MS & [54] \\
\hline Leaves & Tunisia & $\begin{array}{l}\alpha \text {-pinene }(16.8 \%), 4 \text {-terpinenol }(11.9 \%), \beta \text {-phellandrene }(8.9 \%), \\
\text { sabinene }(5.7 \% 9, \gamma \text {-terpinene }(5.5 \%) \text { and } \beta \text {-pinene }(4.3 \%)\end{array}$ & GC-MS & [55] \\
\hline
\end{tabular}


Table 3. Cont.

\begin{tabular}{|c|c|c|c|c|}
\hline Plant Material & Origin & Main Components of Essential Oils or Plant Extracts & Test Assays & Ref. \\
\hline \multirow[t]{3}{*}{ Aerial parts } & $\begin{array}{l}\text { Algeria } \\
\text { (Algiers) }\end{array}$ & $\begin{array}{l}\text { Longifolene }(12.8 \%), \gamma \text {-cadinene }(6.2 \%) \text {, trans- } \beta \text {-terpineol }(5 \%) \text {, } \\
\alpha \text {-acorneol }(4.6 \%), \gamma \text {-muurolene }(4.2 \%), \beta \text {-pinene }(3.7 \%)\end{array}$ & GC, GC-MS & \multirow[t]{3}{*}{ [64] } \\
\hline & $\begin{array}{c}\text { Algeria } \\
\text { (Tizi-Ouzou) }\end{array}$ & $\begin{array}{l}\text { Longifolene }(16.4 \%) \text {, trans- } \beta \text {-terpineol }(15.6 \%) \text {, terpinen- } 4 \text {-ol } \\
(7 \%), \gamma \text {-muurolene }(5.7 \%), \beta \text {-pinene }(3.3 \%), \alpha \text {-pinene }(2.8 \%)\end{array}$ & GC, GC-MS & \\
\hline & Algeria (Oran) & $\begin{array}{c}\alpha \text {-pinene }(19 \%), \text { trans- } \beta \text {-terpineol }(13.1 \%), \text { sabinene }(12.6 \%) \\
\beta \text {-pinene, }(6.5 \%),(\mathrm{E})-\beta \text {-ocimene }(5.5 \%), \text { longifolene }(5.2 \%)\end{array}$ & GC, GC-MS & \\
\hline Leaves & $\begin{array}{l}\text { Sardinia } \\
\text { (Italy) }\end{array}$ & $\begin{array}{c}\alpha \text {-pinene }(14.8-22.6 \%) \text {, terpinen-4-ol }(14.2-28.3 \%), \beta \text {-myrcene } \\
(1.0-18.3 \%), p \text {-cymene }(14.8-16.2 \%), \text { sabinene }(2.5-8.1 \%) \\
\text { limonene }(0.9-3.8 \%)\end{array}$ & GC-MS & [19] \\
\hline Leaves & Greece & $\begin{array}{c}\alpha \text {-pinene }(9.4-24.9 \%), \text { terpinen-4-ol }(6.8-10.6 \%), \mathrm{p} \text {-cymene } \\
(0.5-7.5 \%), \text { limonene }(9.0-17.8 \%), \gamma \text {-terpinene }(3.1-3.6 \%)\end{array}$ & GC-MS & [51] \\
\hline Leaves & $\begin{array}{l}\text { Sardinia } \\
\text { (Italy) }\end{array}$ & $\begin{array}{l}\alpha \text {-pinene, } \alpha \text {-thujene, camphene, sabinene, } \beta \text {-pinene, myrcene, } \\
\alpha \text {-phellandrene, } \alpha \text {-terpinene, para-cymene, } \beta \text {-phellandrene, } \\
\text { trans-ocemene, } \gamma \text {-terpene, terpinolene, } 2 \text {-nonanone, linalool, } \\
\text { isopentyl isovalerate, terpin- } 4 \text {-ol, } \alpha \text {-terpiniol and others. }\end{array}$ & GC-MS & [29] \\
\hline Leaves & Algeria & $\begin{array}{c}\beta \text {-caryophyllene }\left(54-198 \mu \mathrm{g} \mathrm{g}^{-1} \mathrm{dw}\right), \delta \text {-cadinene }(15-186 \mu \mathrm{g} \\
\left.\mathrm{g}^{-1} \mathrm{dw}\right) \text {, cubebol }\left(15-117 \mu \mathrm{g}^{-1} \mathrm{dw}\right), \beta \text {-bisabolene }(22.1-105 \\
\left.\mu \mathrm{g} \mathrm{g}^{-1} \mathrm{dw}\right), \alpha \text {-pinene }\left(1.9-105 \mu \mathrm{g} \mathrm{g}^{-1} \mathrm{dw}\right), \gamma \text {-muurolene } \\
\left(29.7-67.3 \mu \mathrm{g} \mathrm{g}^{-1} \mathrm{dw}\right)\end{array}$ & GC-MS & [65] \\
\hline Leaves & $\begin{array}{l}\text { Sardinia } \\
\text { (Italy) }\end{array}$ & $\begin{array}{c}\text { Germacrene D }(19.9 \%), \beta \text {-caryophyllene }(6.6 \%), \alpha \text {-pinene } \\
(6.3 \%) \text {, myrcene }(3.9 \%), \beta \text {-phellandrene }(3.7 \%), \alpha \text {-humulene } \\
(2.4 \%)\end{array}$ & GC-MS & [12] \\
\hline Leaves & $\begin{array}{l}\text { Eastern } \\
\text { Morocco }\end{array}$ & $\begin{array}{l}\text { Taforalt and Saidia areas: limonene, } \alpha \text {-pinene, } \alpha \text {-terpineol and } \\
\beta \text {-caryophyllene;Laayoune and Jerada areas: myrcene and } \\
\beta \text {-caryophyllene. }\end{array}$ & GC-MS & [66] \\
\hline Fresh leaves & Greece & $\begin{array}{l}\delta \text {-germacrene }(24.78 \%), \text { myrcene }(19.5 \%), \alpha \text {-cadinol }(9.53 \%) \text {, } \\
\gamma \text {-cadinene }(5.59 \%), \text { trans-caryophyllene }(5.03 \%) \text {, limonene } \\
(4.84 \%)\end{array}$ & GC-MS & [67] \\
\hline Dried leaves & & $\begin{array}{c}\delta \text {-cadinene }(17.04 \%), \alpha \text {-amorphene }(10.32 \%), \delta \text {-germacrene } \\
(9.01 \%), \text { trans-caryophyllene }(6.32 \%), \alpha \text {-cubebene }(5.55 \%) \\
\text { naphthalene }(4.13 \%)\end{array}$ & GC-MS & \\
\hline Ripe fruits & Tunisia & Phenolic composition of seed oil (concentrations not shown) & GC-MS & [68] \\
\hline Leaves & Tunisia & $\begin{array}{c}\text { Germacrene D }(11.9 \%) \text {, pinene }(9.9 \%) \text {, limonene }(8.5 \%) \\
\delta \text {-cadinene }(8.5 \%), \beta \text {-caryophyllene }(8.2 \%) \text {, terpinen } 4 \text {-ol }(5.1 \%)\end{array}$ & GC-FID, GC-MS & [69] \\
\hline Fruits & Tunisia & $\begin{array}{c}\alpha \text {-pinene }(13.35 \%), \alpha \text {-phellandrene }(10.12 \%), \beta \text {-phellandrene } \\
(10.45 \%), \text { sabinene }(7.01 \%), \text { germacrene-D }(6.86 \%), \\
\beta \text {-caryophyllene }(4.58 \%)\end{array}$ & GC-MS & [70] \\
\hline Leaves & $\begin{array}{l}\text { Tuscany } \\
\text { (Italy) }\end{array}$ & $\begin{array}{l}\alpha \text {-pinene }(24.6-9.2 \%), 1-4 \text { terpineol }(14.9-7.1 \%), \\
\beta \text {-phellandrene }(11.4-4.7 \%), \beta \text {-pinene }(8.6-1.2 \%), \beta \text {-mircene } \\
(9.2-0.7 \%), \alpha \text {-terpineol }(8.4-4.9 \%)\end{array}$ & GC-MS & [71] \\
\hline Leaves and twigs & $\begin{array}{l}\text { Sardinia } \\
\text { (Italy) }\end{array}$ & $\begin{array}{l}\text { Terpinen-4-ol }(25.2 \%), \alpha \text {-phellandrene }(11.9 \%), \beta \text {-phellandrene } \\
(10.2 \%), \gamma \text {-terpinene }(10.1 \%), \alpha \text {-pinene }(7.6 \%)\end{array}$ & GC-FID, GC-MS & [72] \\
\hline Fruits & Tunisia & $\begin{array}{c}\text { 4-\{3-[(2hydroxybenzoyl }) \text { amino] anilino }\} 4 \text {-oxobut-2-enoic acid } \\
(28.96 \%), \beta \text {-myrcene }(11.47 \%), 3 \text {-pentadecylphenol }(8.51 \%) \\
\text { p-tolyl ester }(8.36 \%), \text { amino formic acid }(7.51 \%)\end{array}$ & GC-MS & [73] \\
\hline Male flowers & Tunisia & $\begin{array}{c}\beta \text {-caryophyllene }(12.8 \%) \text {, germacrene-D }(9.6 \%) \text {, elemol }(8.9 \%), \\
\alpha \text {-terpineol }(7.8 \%), \gamma \text {-cadinene }(7.1 \%), \text { bornyl acetate }(6.2 \%)\end{array}$ & GC-MS & [74] \\
\hline Female flowers & & $\begin{array}{c}\alpha \text {-limonene }(28.7 \%) \text {, germacrene-D }(23.7 \%) \text {, elemol }(6.7 \%), \\
\beta \text {-caryophyllene }(6.6 \%), \alpha \text {-pinene }(6.0 \%), \text { bornyl acetate }(3.7 \%)\end{array}$ & GC-MS & \\
\hline
\end{tabular}


Table 3. Cont.

\begin{tabular}{|c|c|c|c|c|}
\hline Plant Material & Origin & Main Components of Essential Oils or Plant Extracts & Test Assays & Ref. \\
\hline $\begin{array}{l}\text { Leaves of male } \\
\text { plants at flowering }\end{array}$ & & $\begin{array}{l}\alpha \text {-limonene }(18.8 \%), \text { germacrene-D }(13.1 \%), \beta \text {-caryophyllene } \\
(8.8 \%), \delta \text {-cadinene }(8.7 \%), \gamma \text {-cadinene }(6.2 \%), \alpha \text {-pinene }(4.8 \%)\end{array}$ & GC-MS & \\
\hline $\begin{array}{l}\text { Leaves of female } \\
\text { plants at flowering }\end{array}$ & & $\begin{array}{l}\text { Germacrene-D }(20.7 \%), \delta \text {-cadinene }(15.6 \%), \beta \text {-caryophyllene } \\
(12.1 \%), \gamma \text {-cadinene }(6.6 \%), \delta \text {-cadinol }(6.1 \%), \alpha \text {-limonene }(5 \%)\end{array}$ & GC-MS & \\
\hline Ripe fruits & & $\begin{array}{c}\beta \text {-myrcene }(75.6 \%), \alpha \text {-pinene }(12.6 \%), \alpha \text {-limonene }(3.2 \%) \\
\alpha \text {-terpineol }(1.4 \%), \text { camphene }(0.8 \%)\end{array}$ & GC-MS & \\
\hline Leaves & Morocco & $\begin{array}{c}\text { Myrcene (33.5\%), } \alpha \text {-pinene (19.2\%), limonene }(6.6 \%) \\
\alpha \text {-phellandrene }(4.6 \%), \gamma \text {-terpineol }(3.7 \%), \alpha \text {-terpineol }(3.6 \%)\end{array}$ & GC-MS & B \\
\hline Leaves & $\begin{array}{l}\text { Sardinia } \\
\text { (Italy) }\end{array}$ & $\begin{array}{c}\alpha \text {-pinene }(16.9 \%), \text { terpinen-4-ol }(16.5 \%), \text { sabinene }(7.8 \%) \\
\alpha \text {-phellandrene }(7.4 \%), \gamma \text {-terpinene }(6.3 \%), \beta \text {-pinene }(4.3 \%)\end{array}$ & GC-MS & [49] \\
\hline \multicolumn{5}{|l|}{$\begin{array}{l}\text { Plant } \\
\text { extracts/solvent } \\
\text { used }\end{array}$} \\
\hline $\begin{array}{l}\text { Leaves/ethyl } \\
\text { acetate and } \\
\text { methanol }\end{array}$ & Italy & $\begin{array}{c}\text { 3,5-O-digalloyl quinic acid }(26.8 \pm 0.15 \mathrm{mg} / \mathrm{g} \mathrm{DW}) \\
\text { 3,4,5-O-trigalloyl quinic acid }(10.3 \pm 2.45 \mathrm{mg} / \mathrm{g} \mathrm{DW}), 5-\mathrm{O}- \\
\text { galloyl quinic acid }(9.6 \pm 2.45 \mathrm{mg} / \mathrm{g} \text { DW }), \text { myricetin } \\
\text { 3-O-rhamnoside }(6.8 \pm 1.04 \mathrm{mg} / \mathrm{g} \text { DW }) \text { myricetin } \\
\text { 3-O-rutinoside }(4.5 \pm 0.18 \mathrm{mg} / \mathrm{g} \mathrm{DW}), \text { myricetin glucuronide } \\
(3.9 \pm 0.65 \mathrm{mg} / \mathrm{g} \mathrm{DW})\end{array}$ & $\begin{array}{l}\text { HPLC-DAD, } \\
\text { HPLC-MS, } \\
\text { NMR }\end{array}$ & [60] \\
\hline Berries/methanol & Apulia (Italy) & $\begin{array}{l}\text { Cyanidin 3-O-glucoside (71\%), delphinidin 3-O-glucoside, } \\
\text { cyanidin 3- O arabinoside }(28-31 \%)\end{array}$ & $\begin{array}{l}\text { HPLC-DAD- } \\
\text { MS }\end{array}$ & [76] \\
\hline $\begin{array}{l}\text { Fruits during matu- } \\
\text { ration/petroleum } \\
\text { ether }\end{array}$ & Tunisia & Oils, fatty acids and sterols & GC-MS & [35] \\
\hline Leaves/methanol & Algeria & $\begin{array}{l}46 \text { compounds (most abundant flavonoids, phenolic acids and } \\
\text { their derivatives) }\end{array}$ & $\begin{array}{l}\text { HPLC-ESI- } \\
\text { QTOF }\end{array}$ & [61] \\
\hline Leaves/methanol & Italy & 46 secondary metabolites & LC-ESI-MS/MS & [77] \\
\hline $\begin{array}{l}\text { Fruits/methanol- } \\
\text { water }\end{array}$ & Tunisia & $\begin{array}{c}\text { Total phenolic acids } 436.4-2762.7 \mathrm{mg} / \mathrm{kg} \text {; total flavones } \\
75.3-1222.9 \mathrm{mg} / \mathrm{kg} \text {; total flavonols } 24.2-377.4 \mathrm{mg} / \mathrm{kg} \text {; total } \\
\text { secoiridoids } 12.6-366.8 \mathrm{mg} / \mathrm{kg} \text {; total phenols } 538.0-4260.6 \\
\mathrm{mg} / \mathrm{kg}\end{array}$ & $\begin{array}{l}\text { HPLC- } \\
\text { DAD/MSD }\end{array}$ & [78] \\
\hline Leaves/ethanol & Italy & $\begin{array}{c}\text { Tannin derivatives }(70.5 \%) \text {, myricetin derivatives }(22 \%) \text {, } \\
\text { quercetin derivatives }(7.2 \%)\end{array}$ & HPLC-DAD & [79] \\
\hline Leaves/methanol & Egypt & $\begin{array}{l}\alpha \text {-pinene }(38.1 \%), 3,5-\mathrm{O} \text {-digalloyl quinic acid }(13.5 \%), \\
\text { D-limonene }(11.9 \%), \alpha \text {-phellandrene }(10.1 \%), \beta \text {-pinene }(9.5 \%), \\
\gamma \text { muurolene }(8.0 \%) \text {, luteolin-3-O-rutinoside }(7.8 \%) \text {, quercetin } \\
\text { 3-O-di-hexose O-pentose }(7.6 \%), 3,4,5-O \text {-trigalloyl quinic acid } \\
(6.1 \%) \text {, quercetin 3-O-glucuronide }(4.6 \%) \text {, epicatechin 3-gallate } \\
(4.5 \%) \text {, camphene }(3.8 \%)\end{array}$ & $\begin{array}{l}\text { UHPLC-ESI- } \\
\text { MS, } \\
\text { GC-MS }\end{array}$ & [80] \\
\hline
\end{tabular}

Among the compounds in PIL extracts there were discovered to be relevant antioxidant agents, which may attest not only to the activity of PIL in preventing diabetic complications, cholesterol absorption and lipid metabolism [81,82], but also the remarkable capacity of $\mathrm{PIL}$ in managing intestinal inflammatory diseases as reported in the ethnopharmacological survey (Table 2). In fact, recent studies validated the capacity of polyphenols in managing the microbial and metabolomic patterns in the body [83-86]. Particularly, at the intestinal tract, these compounds can stimulate the multiplication of beneficial microorganisms and prevent the adhesion or directly disrupt the membrane ions flux of pathogens $[87,88]$.

\section{Anti-Inflammatory and Antioxidative Activities}

As mentioned above, the anti-inflammatory effect of PIL is of high relevance in ethnopharmacology. The presence of important anti-inflammatory terpenes in the com- 
position of PIL EO can explain its efficacy. It is well-demonstrated that terpenes are capable of inhibiting several inflammatory molecules, e.g., IL-1 $\beta$, IL-6, TNF $\alpha$ and COX$2[6,7,10]$, thus disrupting the amplification of inflammatory mechanisms. Meanwhile, the anti-inflammatory properties of PIL extracts can be related to the richness of polyphenols, the interplay of which in the inflammatory cascade is mainly demonstrated toward macrophages by inhibiting multiple key regulators of the inflammatory response [89]. Additionally, polyphenols reduce the release of arachidonic acid, prostaglandins and leukotrienes directly related to the inhibition of COX and LOX [90]. Other considerations regard several flavonoids in polyphenols, which can directly modulate the expression of pro-inflammatory cytokines and chemokines [91]. The ability of these natural compounds to modify the expression of several pro-inflammatory genes in addition to their antioxidant characteristics, such as reactive oxygen species (ROS) scavenging, contributes to the regulation of inflammatory signaling [92,93].

To clarify scientifically the anti-inflammatory character of PIL EO and extracts, they have undergone investigations by in vitro and in animal model studies with the intent to elucidate any selective interaction toward proteins and enzymes participating in the inflammatory pathway (Table 4).

Table 4. Antioxidant and anti-inflammatory activity of Pistacia lentiscus.

\begin{tabular}{|c|c|c|c|c|c|c|}
\hline Exp. Setting & Origin Model & Plant Material & Model & Exp. Protocol & Results & Ref. \\
\hline \multirow{11}{*}{$\begin{array}{l}\text { Antioxidant } \\
\text { activity }\end{array}$} & Sardinia, Italy & Leaves oil & Cells free & $\begin{array}{l}\text { DPPH as Trolox equivalent } \\
\text { antioxidant capacity (TEAC) }\end{array}$ & $\begin{array}{c}\text { Great seasonal } \\
\text { variability inhibition }\end{array}$ & [19] \\
\hline & Algeria & Leaves extract & Cells free & $\begin{array}{c}\text { FRAP } \\
\mathrm{H}_{2} \mathrm{O}_{2} \text { scavenging activity }\end{array}$ & $\begin{array}{l}\uparrow \text { High } \\
\downarrow \text { Low }\end{array}$ & [86] \\
\hline & Algeria & Leaves extract & Cells free & $\begin{array}{c}\text { Ferric reducing antioxidant power } \\
\text { (FRAP) } \\
\text { DPPH } \\
\mathrm{H}_{2} \mathrm{O}_{2} \text { scavenging activity } \\
\text { Linoleic acid peroxidation } \\
\text { inhibition }\end{array}$ & $\begin{array}{l}\uparrow \text { High and dose } \\
\text { dependent } \\
\uparrow \uparrow \text { Very high } \\
\uparrow \uparrow \text { Very high } \\
\uparrow \uparrow \uparrow \text { Outstanding }\end{array}$ & [94] \\
\hline & $\begin{array}{l}\text { Zakynthos } \\
\text { (Greece) }\end{array}$ & Leaves extract & Cells free & $\begin{array}{l}\text { DPPH } \\
\text { Ferric reducing antioxidant power } \\
(\text { FRAP) }\end{array}$ & $\begin{array}{l}\uparrow \uparrow \text { Very high } \\
\uparrow \text { High }\end{array}$ & [51] \\
\hline & Sardinia, Italy & Leaves extract & Cells free & $\begin{array}{l}\text { DPPH as Trolox equivalents } \\
\text { ABTS as Trolox equivalents }\end{array}$ & $\begin{array}{l}\uparrow \uparrow \text { High } \\
\uparrow \uparrow \text { High }\end{array}$ & [95] \\
\hline & Algeria & Leaves extract & Cells free & $\begin{array}{l}\text { DPPH }(\%) \\
\text { Ferric reducing antioxidant power } \\
\text { (FRAP) } \\
\beta \text {-carotene bleaching method }(\%)\end{array}$ & $\begin{array}{l}\uparrow \text { High } \\
\uparrow \text { High } \\
\uparrow \uparrow \text { Very high }\end{array}$ & [35] \\
\hline & Morocco & $\begin{array}{l}\text { Fruits oil, } \\
\text { leaves oil }\end{array}$ & Cells free & $\begin{array}{l}\text { DPPH } \\
\text { FRAP } \\
\text { ABTS }\end{array}$ & $\begin{array}{l}\text { Fruits oil: } \uparrow \uparrow \text { high } \\
\text { Leaves oil: } \uparrow \text { high } \\
\text { Fruits oil: } \uparrow \uparrow \text { high } \\
\text { Leaves oil: } \uparrow \text { high } \\
\text { Fruits oil: } \uparrow \uparrow \text { high } \\
\text { Leaves oil: } \uparrow \text { high }\end{array}$ & [75] \\
\hline & $\begin{array}{l}\text { Campania } \\
\text { (Italy) }\end{array}$ & Leaves extract & Cell lines & $\begin{array}{l}\text { Lipid peroxidation } \\
\text { Intracellular ROS } \\
\text { Oxidized glutathione }\end{array}$ & $\begin{array}{l}\uparrow \uparrow \text { Very high } \\
\uparrow \uparrow \text { Very high } \\
\uparrow \uparrow \text { Very high }\end{array}$ & [15] \\
\hline & Sardinia, Italy & Leaves oil & Animals & DHA & $\uparrow \uparrow$ High protection & [12] \\
\hline & Algeria & $\begin{array}{l}\text { Fruits extract, } \\
\text { leaves extract }\end{array}$ & $\begin{array}{l}\text { Cells free and } \\
\text { cell lines }\end{array}$ & $\begin{array}{l}\text { Intracellular ROS in THP-1 } \\
\text { monocytic cells }\end{array}$ & $\begin{array}{c}\text { Fruits extract: } \\
\text { dose-dependent } \\
\text { protection } \\
\text { Fruits extract: } \\
\uparrow \text { High; } \\
\text { Leaves extract: } \\
\uparrow \uparrow \text { Very high }\end{array}$ & [96] \\
\hline & Sardinia, Italy & Leaves oil & $\begin{array}{l}\text { Cells free and } \\
\text { cell lines, } \\
\text { human } \\
\text { fibroblasts }\end{array}$ & $\begin{array}{c}\mathrm{H}_{2} \mathrm{O}_{2} \text { scavenging activity } \\
\text { ECC }\end{array}$ & $\begin{array}{l}\downarrow \text { Low } \\
\downarrow \text { Low }\end{array}$ & [49] \\
\hline
\end{tabular}


Table 4. Cont.

\begin{tabular}{|c|c|c|c|c|c|c|}
\hline Exp. Setting & Origin Model & Plant Material & Model & Exp. Protocol & Results & Ref. \\
\hline \multirow{4}{*}{$\begin{array}{l}\text { Anti- } \\
\text { inflammatory } \\
\text { activity }\end{array}$} & Sardinia, Italy & Leaves oil & Animals & COX-2 & $\uparrow \uparrow$ High inhibition & [12] \\
\hline & Sardinia, Italy & Leaves oil & Animals & $\begin{array}{l}\text { TNF- } \alpha \\
\text { IL-6 }\end{array}$ & $\begin{array}{l}\downarrow \downarrow \text { High decrease } \\
\downarrow \downarrow \downarrow \text { High decrease }\end{array}$ & [29] \\
\hline & Algeria & $\begin{array}{l}\text { Fruits extract, } \\
\text { leaves extract }\end{array}$ & $\begin{array}{l}\text { Cells free and } \\
\text { cell lines }\end{array}$ & $\begin{array}{l}\text { IL-1 } \beta \text { inhibition by ATP } \\
\text { stimulated THP-1 } \\
\text { IL-1 } \beta \text { inhibition by } \mathrm{H}_{2} \mathrm{O}_{2} \\
\text { stimulated THP-1 }\end{array}$ & $\begin{array}{c}\text { Fruit extract: } \\
\text { no reduction; } \\
\text { Leaves extract: } \\
\uparrow \uparrow \text { high } \\
\text { Fruit extract: } \\
\downarrow \text { low; } \\
\text { Leaves extract: } \\
\text { dose-dependent }\end{array}$ & [96] \\
\hline & Sardinia, Italy & Leaves oil & $\begin{array}{l}\text { Cells free and } \\
\text { cell lines, } \\
\text { Human } \\
\text { fibroblasts }\end{array}$ & $\begin{array}{l}\text { COX-1 } \\
\text { COX-2 } \\
\text { LOX }\end{array}$ & $\begin{array}{c}\text { Inhibition } \\
\uparrow \text { high inhibition } \\
\text { no inhibition }\end{array}$ & [49] \\
\hline
\end{tabular}

DPPH = 2,2-diphenyl-1-picryl-hydrazyl-hydrate assay; FRAP = ferric reducing activity power assay; MTT = 3-(4,5-dimethylthiazol2-yl)-2,5-diphenyltetrazolium bromide; ABTS = 2,20-azinobis (3-ethyl- benzothiazoline-6-sulphonic acid) diammonium salt; WST-8 = (2-(2-methoxy-4-nitrophenyl)-3-(4-nitrophenyl)-5-(2,4-disulfophenyl)-2H-tetrazolium, monosodium salt) assay; IC50 = percentage of cytotoxicity and concentration which inhibit half-cell population compared to the drug-free control; CC50 = concentration which inhibits cell metabolism by $50 \%$ compared to the drug-free control; DHA $=$ docosahexaenoic acid; $\mathrm{BCB}=\beta$-carotene bleaching test; $\mathrm{ECC}=$ electro-chemical characterization assay; $\mathrm{DCF}=2^{\prime}, 7^{\prime}$ dichlorofluorescein diacetate; SRB = sulforhodamine $\mathrm{B} ; \mathrm{ORAC}=\mathrm{oxygen}$ radical absorbance capacity; IL-1 $\beta=$ interleukin-1 $\beta$; IL-6 = interleukin-6; TNF- $\alpha=$ tumor necrosis factor- $\alpha$; COX-1 = cyclooxygenase 1 ; COX-2 = cyclooxygenase 2 ; and LOX = lipoxygenase.

\subsection{Inhibitory Activity against Proinflammatory Cytokines and against Arachidonic Acid Cascade}

Remila and co-workers [96] examined the anti-inflammatory activity of leaves and fruits extracts by measuring the secretion of IL- $1 \beta$ by macrophages exposed to adenosin triphosphate (ATP) or $\mathrm{H}_{2} \mathrm{O}_{2}$. The authors found PIL leaves extract significantly reduced the production of IL-1 $\beta$ from ATP- or $\mathrm{H}_{2} \mathrm{O}_{2}$-activated cells. The inhibitory capacity of the leaves extract was higher in comparison to that of the fruits and of quercetin and gallic acid (tested as isolated fractions of the polyphenol mixture). The data was explained by the higher content of the total phenols and flavonoids in the leaves compared to the fruits and by the synergy between the pharmacological biomolecules of PIL extract. Similar considerations and a dose-dependent anti-inflammatory effect of the leaves extract was reported in other studies, which further strengthened the capacity of flavonoids and tannins [97].

Comparable anti-inflammatory values were reported in regard to PIL EO using the carrageenan-induced paw edema and cotton pellet-induced granuloma in a rat model [29]. Particularly, it was evidenced that when applied topically, PIL EO from leaves significantly inhibited the development of granuloma and the serum level of TNF- $\alpha$ and IL- 6 in reply to the irritants. The result was mainly related to the activity of $\alpha$-pinene, $\beta$-pinene, $\alpha$ - phellandrene and sabinene, which were highly represented in the chemistry of the hydrodistilled oil.

Only one study has investigated the inhibitory activity of the whole PIL EO. It was obtained from the leaves of plants growing in Sardinia and tested against COXs and LOX [49]. The $\mathrm{IC}_{50}$ values were $10.3 \pm 4.4 \mu \mathrm{g} / \mathrm{mL}$ and $6.1 \pm 2.5 \mu \mathrm{g} / \mathrm{mL}$ PIL EO for COX-1 and COX-2, respectively, with higher inhibitory activity toward COX-2 in comparison to that produced towards COX-1. Additionally, COX-2 inhibition by the EO was similar to that recorded using ibuprofen as a positive control. The activity of the oil against LOX did not reach the IC 50 value, as PIL EO lowered LOX activity by $30 \%$ compared to the control. Despite the low LOX inhibition, the study strengthens the oil as a potential dual inhibitory compound, which was intensively researched in pharmacology to antagonize a great number of inflammatory processes where these enzymes sustain and amplify the disease [68,78]. The data obtained in that investigation were addressed to the mixture of terpenoids comprising the oil, with particular regard to $\alpha$-pinene and terpinen-4-ol $(33.38 \%)$, enriched by the non-cannabinoid terpenoid limonene (3.4\%), $\beta$-myrcene $(0.9 \%)$, (Z)-caryophyllene 
(1.4\%) and (E)- $\beta$-caryophyllene (0.1\%). The mixture allowed the classification of the oil as pharmacologically active [50].

\subsection{Inhibiting Activity against ROS Molecules}

The protective effect of PIL EO and extracts against ROS has been deeply documented in the literature (Table 5). From a scientific point of view, this capacity can be related to the terpenes and polyphenols of the $\mathrm{EO}$ and of the extracts, respectively [10,59]. As the accumulation of ROS directly affects the healthy tissue systems, including cellular lipids, nucleic acids and proteins [98], the capacity of the EO and the extracts was studied directly using cell lines and intracellular ROS evaluation assays, or by chemical methods, particularly using the 2,20-azinobis(3-ethylbenzothiazoline-6-sulphonic acid) diammonium salt (ABTS) and the 1,1-diphenyl-2- picrylhydrazyl (DPPH) chemical assays, and more recently using electron chemical devices.

As it is shown in Table 4, the anti-ROS ability has been mainly investigated in PIL extracts. Scientifically, this ability has been addressed to the richness in polyphenols the extracts possess, which not only inhibits the production of ROS by the direct involvement of specific molecules [99], but also can modulate the Keap1-Nrf2/ARE pathway [100]. This is a powerful oxidation-reduction defense system, where polyphenols act to degrade specifically the Keap1 protein and regulate the Nrf2-related pathway $[59,101]$.

Remila and co-workers [96] proved the high antioxidant capacity of PIL leaves extract using the oxygen radical absorbance capacity in macrophages, melanoma and mammary mouse cell lines. Furthermore, due to the activation of apoptosis mechanisms, the extracts significantly inhibited the growth of melanoma cells. The data was related to the richness in phenolics, flavonoids and tannins in the extracts. Similar conclusions were obtained by Atmani [94], who examined hexane and chloroform aqueous extracts of PIL leaves containing highly concentrated flavonoids. In relation to the peak of hydroxyl groups, the aqueous formulations strongly inhibited lipid peroxidation. The mechanism was explained by the scavenging of peroxyl radicals by the extracts. Radicals play a role in the development of cardiovascular disease and cancer. In this regard, the scavenger activity of gallic acid and galloylquinic derivatives, isolated from PIL leaves, is particularly attractive. Notably, a progressive increase in the anti-radical activity associated with the number of galloyl groups in quinic acid was found [102]. Furthermore, all the tested metabolites strongly reduced the oxidation of low-density lipoproteins, thus strengthening the protection of PIL against the lipid peroxidation.

In addition to having a preventive capacity, a potential ability to halt or reverse oxidative stress-related diseases has been attributed to PIL. In fact, the ability to fight aggressive tumors, i.e., neuro-blastoma [19], and other important tumor cell lines has been proved by in vitro studies $[103,104]$.

Animal testing further explored the anti-ROS efficacy of PIL derivates. Ben Khedir and co-workers [70] determined the scavenger and anti-inflammatory activity of the fruits oil using the carrageenan-induced paw edema in a rat model. In that study, PIL oil demonstrated significantly better anti-inflammatory activity with edema inhibition, in comparison to those produced by the control NADPH. Moreover, PIL oil was able to increase the expression of superoxide dismutase, catalase and glutathione peroxidase, which are released as a response to the oxidative stress in the inflamed tissue. The effects were interpreted as a consequence of the content of humulene, caryophyllene and polyunsaturated fatty acid in the oil. Humulene and caryophyllene have been shown to inhibit the nuclear factor kappa B (NF-kB) pathway, responsible for the transcription of several proinflammatory cytokines, i.e., TNF- $\alpha$, IL-1 $\beta$, IL- 6 and iNOS and COX-2 enzymes [105]. The polyunsaturated fatty acid in the oil might have partially replaced the arachidonic acid in the inflamed cell membranes [57], consequently lowering COX-2 production, the local inflammation and ROS generation.

Other in vivo studies demonstrated that an administration of PIL oil before the induction of the Bilateral Common Carotid Artery Occlusion followed by Reperfusion (BC- 
$\mathrm{CAO} / \mathrm{R}$ ) was able to prevent the oxidative stress challenge in the nervous tissue due to the ischemic insult $[12,106]$. In the cerebral tissue, PIL oil restored the membrane phospholipid DHA and decreased the activity of the COX-2 enzyme. Additionally, PIL oil increased the concentration of the anti-inflammatory endocannabinoid congeners palmitoylethanolamide (PEA) and oleoylethanolamide (OEA) [12]. The outcomes were related to the high presence of the phytocannabinoid (E)- $\beta$-caryophyllene, which worked synergistically with the other compounds in the oil, expanding the levels of cannabinoid receptor type 2 (CB2) and PPAR-alpha receptors. Further studies attest to the role of $\beta$-caryophyllene as CB2 agonists, demonstrating its capacity to antagonize the release of cytokines from LPS-stimulated monocytes (TNF- $\alpha$ and IL-1 $\beta$ ) [7].

Table 5. (a) Antibacterial activity of Pistacia lentiscus L. determined by agar diffusion test (ADD) or minimal inhibitory concentration (MIC). (b) Antifungal activity of Pistacia lentiscus L. determined by agar diffusion test (ADD) or minimal inhibitory concentration (MIC).

(a)

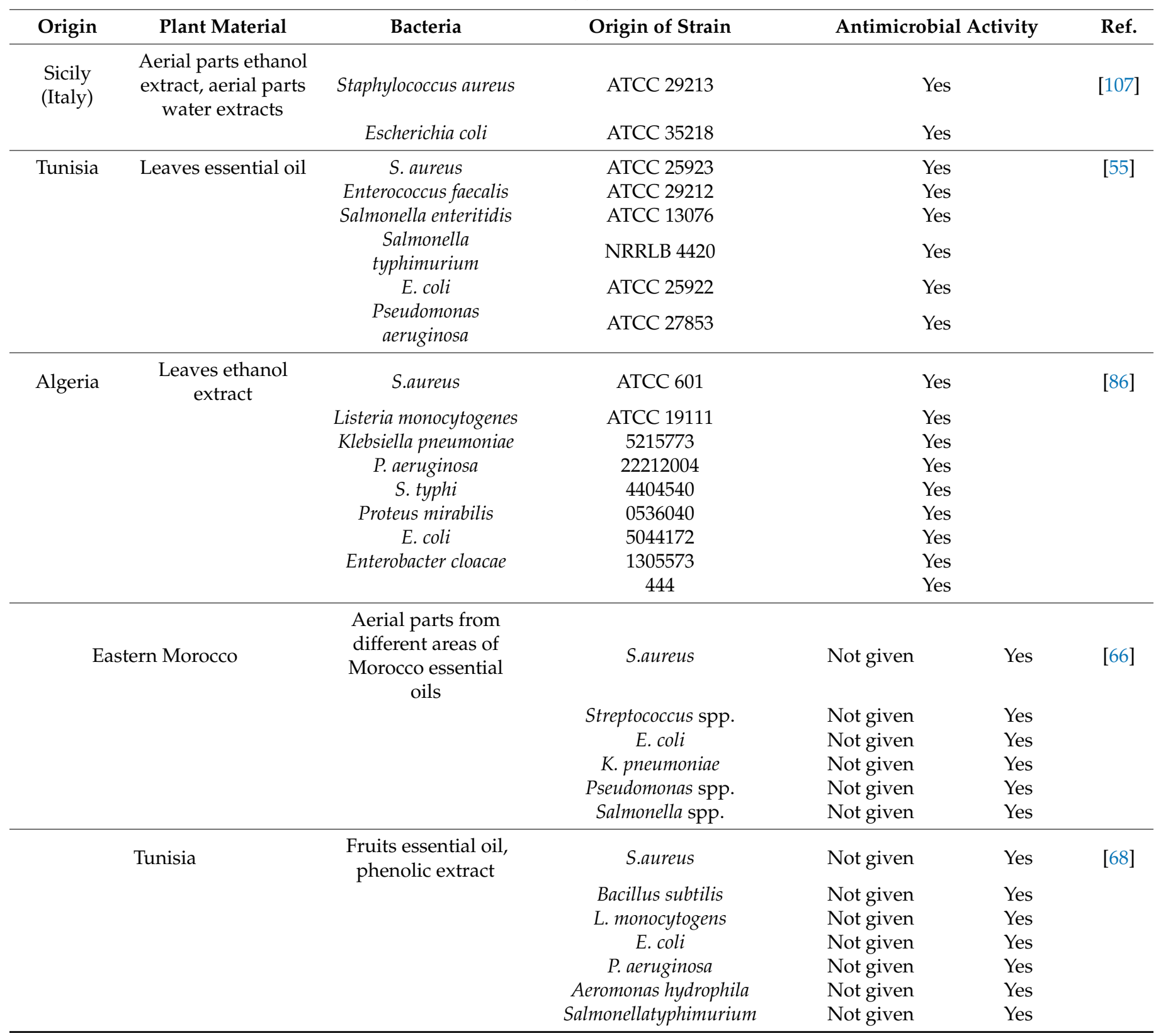


Table 5. Cont.

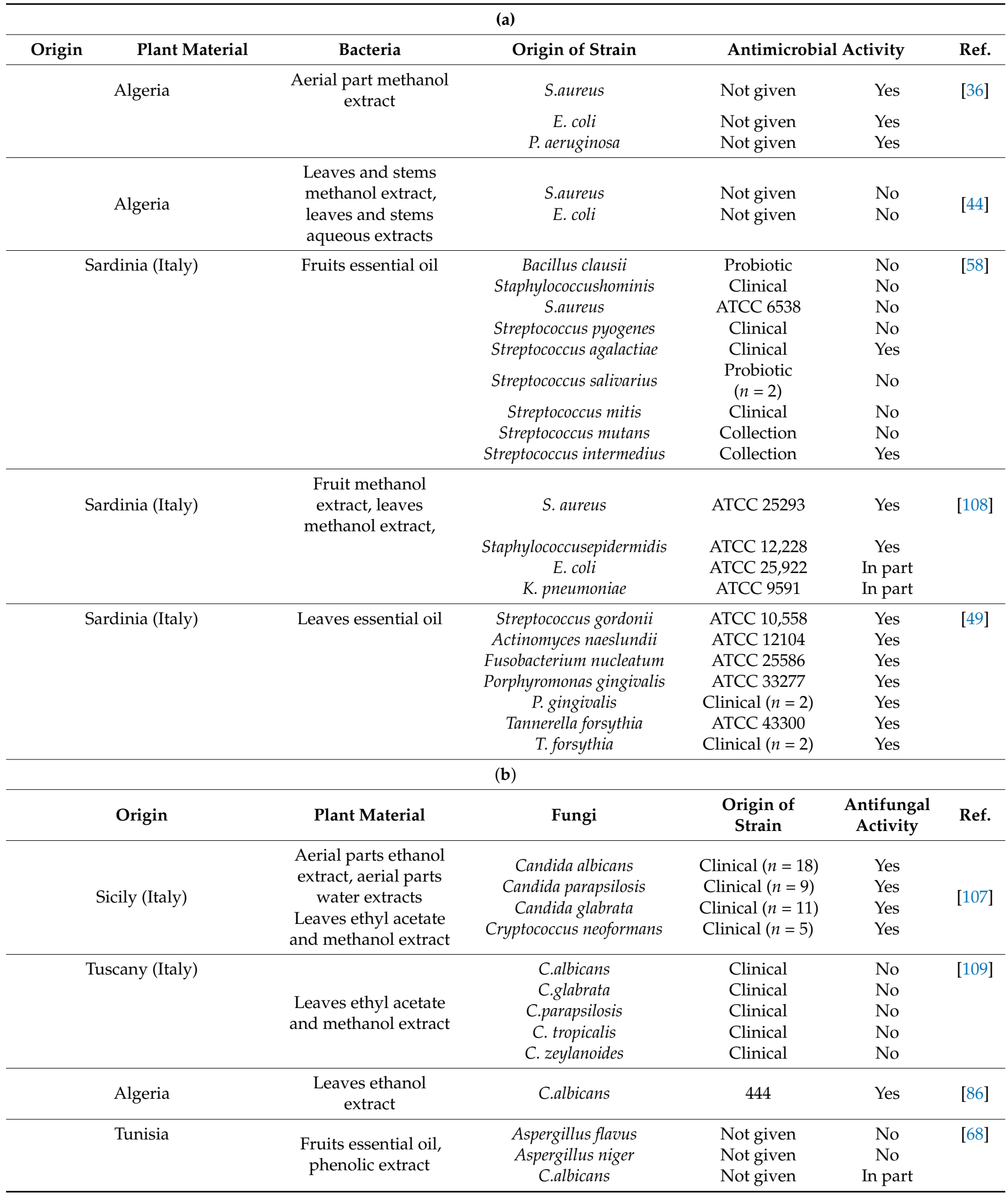


Table 5. Cont.

\begin{tabular}{|c|c|c|c|c|c|}
\hline \multicolumn{6}{|c|}{ (b) } \\
\hline Origin & Plant Material & Fungi & $\begin{array}{l}\text { Origin of } \\
\text { Strain }\end{array}$ & $\begin{array}{c}\text { Antifungal } \\
\text { Activity }\end{array}$ & Ref. \\
\hline \multirow{3}{*}{ Sardinia (Italy) } & \multirow{3}{*}{ Fruits essential oil } & C. albicans & Clinical & No & \multirow[t]{3}{*}{ [58] } \\
\hline & & C.glabrata & Clinical & No & \\
\hline & & C.krusei & Clinical & No & \\
\hline \multirow[t]{4}{*}{ Sardinia (Italy) } & \multirow[t]{4}{*}{ Leaves essential oil } & C.albicans & Laboratory & Yes & \multirow[t]{4}{*}{ [49] } \\
\hline & & C.albicans & Clinical $(n=2)$ & Yes & \\
\hline & & C.glabrata & Laboratory & Yes & \\
\hline & & C.glabrata & Clinical $(n=2)$ & Yes & \\
\hline
\end{tabular}

\section{Potential Cytotoxicity}

Starting from Paracelsus' statement "the right dose differentiates a poison to a remedy", investigations have been conducted with the intent to validate pharmaceutically PIL derivatives. For this reason, many studies have been conducted, in particular using in vitro and cell lines models testing different doses of PlL oil and extracts (Table 4). As a measure of risk versus benefit, many of them also applied enzymes testing, looking for the capacity of the EO and extracts to interact with the proteins and their involvement in inflammation and oxidative stress. In fact, it is well-known that the in vitro and cell lines systems are largely recommended to elucidate the safety of herbal products and propose the natural agents as nutraceuticals or xenobiotics [110]. Although such models lack the complexity of animals, and the compounds in testing should not exert in vivo the same effects as reported in isolated cell tissues [111], they have a significant role in predicting of risks and toxicology.

As a result of this work, it can be stated that not any published laboratory study reported cytotoxic effects which could be connected to PIL oil or extracts. Conversely, the experiments demonstrated direct or indirect biocompatibility of PIL derivatives to humans and non-human animals.

Notably, a wide range of biocompatibility was documented in oral cells. It was remarked specifically in oral human fibroblast cell lines using the EO from leaves and the MTT reduction assay [49]. That fact was further validated in the periodontal ligament fibroblasts, the gingival fibroblasts, the gingival keratinocytes and dysplastic oral keratinocytes applying the WST-1 metabolic activity assay [49].

The absence of side effects to oral cells is strengthened concerning the polyphenol extracts. In detail, the fruits extract showed high biocompatibility and a selecting index (SI) of cytotoxicity equal to $>256$ toward the human gingival cells, at the same time demonstrating a strong response against periodontal bacteria [112].

\section{Antimicrobial Activity}

Several studies reported the antimicrobial activity of PIL oils and extracts, trying to clarify scientifically their popular use in infectious diseases.

Commonly studied pathogens comprise bacteria known for antibiotic resistance (Staphylococcus aureus incl. methicillin-resistant strains (MRSA), Escherichia coli and Pseudomonas aeruginosa), and other bacteria associated with the oral diseases, as well as yeasts, with particular regard to Candida albicans (Table 5a,b).

Different antimicrobial activity was reported in the studies testing PIL EO and extracts. A high capacity against both bacteria and yeasts was demonstrated in regard to the leaves EO from plants growing in different regions $[16,57,67]$. Whereas the fruits EO from Tunisia [68] and Sardinia [58] were found to have a limited effect against bacteria and yeasts, the ethanol and water extracts of leaves from plants growing in Sicily inhibited the growth of S. aureus, E. coli and yeasts [107]. Additionally, the leaves ethanol or methanol extracts showed activity against several Gram-positive and Gram-negative bacteria $[38,87,113]$. 
However, leaves and stems methanolic or water extracts prepared in Algeria were inactive against S. aureus and E. coli [44], and leaves alcoholic extracts from Tuscany did not act against yeasts [109].

The research started from the fact that terpenoids in EO exert a wide-spectrum of antibacterial, antifungal and even anti-viral activity, and have been demonstrated to inhibit the growth of drug-resistant microbial strains, which are difficult to be treated even by conventional antibiotics [113]. Although $\alpha$-pinene together with the monoterpenes terpinene and myrcene are among the most represented fractions in the Mediterranean oils showing antimicrobial capacity (Table 5), it is of general interest if higher activity could be related to specific PIL chemotypes [114]. In this context, as reported regarding the anti-inflammatory capacity, synergy between the chemical fractions of terpenes is proposed to explain PIL EO antimicrobial activities. Synergy was recently claimed to explain the inhibitory activity of the EO against Porphyromonas gingivalis, Tannerella forsythia and Fusobacterium nucleatum [49]. The result was related to the pharmacological interplay of the terpenes characterizing the oil chemotype from North Sardinia, which was $\alpha$-pinene-terpinen-4$\mathrm{ol}$, further augmented by the non-cannabinoid terpenoids limonene and $\beta$-myrcene, the sesquiterpenes (Z)-caryophyllene and (E)- $\beta$-caryophyllene. Similarly, the antimicrobial efficacy of extracts can be attributed to the richness of polyphenols $[25,38,46,69,70,76,111]$, in particular in regard to the concentrations of tannins, flavonoids, and lignin-carbohydrate complexes in the polyphenols mixture [11]. This is also the case with the ethanol extract of fruits, which showed the highest inhibition potential against $P$. gingivalis in comparison to 20 other extracts of pharmaceutical plants [112]. Furthermore, the potency of the fruits extract were higher than that of the leaves and woody parts, with MIC values against P. gingivalis of $8 \mu \mathrm{g} / \mathrm{mL}$.

Recently, Mandrone and co-workers [108] related the antimicrobial activity of aqueous $\mathrm{MeOH}$ extracts of fruits and leaves to the concentration of phenolic components. That research further attested to the fact that phenols are active against multi-resistant bacteria, among them MRSA and carbapenemase-producing Klebsiella pneumoniae.

Concerning Candida spp., the activity of PIL derivates is reportedly controversial (Table 5). Low or no susceptibility of the yeasts to the leaves extract, or to the fruits oil, was found. Despite this, PIL leaves EO from Sardinia had low MIC values against C. glabrata and C. albicans [49]. The results were explained as a consequence of the recurrence of pharmacological concentrations of six terpenes, which were above $0.05 \%$ in the fingerprint. Additionally, the EO documented the ability to inhibit COX-2 and LOX, which are very important proteins for the development of Candida virulence [115].

Furthermore, a high inhibition of C. albicans was reported when using PIL extracts. The activity of water or ethanol extracts has been attributed to the flavonoid contents. Notably, the phenolic compound tannic acid was reported as more active against the yeast than the antifungals nystatin and amphotericin $[68,75]$.

\section{Summary and Conclusions}

In this review, we summarized the existing knowledge about PIL phytochemistry and some of its biological activities, mainly focusing on its anti-inflammatory and antimicrobial capacity.

The chemistry shows that PIL EO is composed of up to 64 molecules, while 46 constituents have been identified in the extracts. Further minor fractions were determined in the reports analyzing both the $\mathrm{EO}$ and the extracts even if they could not be quantified. The more recurrent chemical components in the $\mathrm{EO}$ from plants growing in the Mediterranean area are represented by $\alpha$-pinene, terpinenes, caryophyllene, limonene and myrcene. Important properties in antagonizing immune-mediated and autoimmunity, neuro-inflammatory, neurological and neurodegenerative diseases, in addition to infections and cancer have been addressed to these molecules. However, the biological character of PIL cannot be entirely focused on one of the main concentrated molecules. The abilities should be attributed to the whole mixture of the terpenes working in synergy, 
or in addition independently by their concentration in the agent. It is remarkable to note that concentrations of non-cannabinoid terpenoids equal to or above $0.05 \%$ increase the pharmacological potency of PIL oil.

Regarding the extracts, the high polyphenol content is attractive for prevention, and for therapy of chronic illness and infections. The richness in polyphenols suggests the use of the extracts as nutraceuticals in human health. Among the compounds, flavonol glycosides, i.e., myricetin and quercetin glycosides, could indicate a possible role of PIL in preventing diabetic complications and managing intestinal inflammatory response. Meanwhile, the concentration of bioactive flavanones should put forward the extracts to manage cholesterol absorption, glucose and lipid metabolism. Furthermore, the quinic acid, lignans and anthocyanins content is attractive in view of the high antioxidant capacity, in particular when water-extracted from PIL.

Nevertheless, although useful information has been provided in in vitro and animal model studies, clinical trials are necessary to fully understand the capacity and limitations of PIL EO and extracts in humans.

Regarding the anti-inflammatory capacity, PIL EO and extracts are able to inhibit the proinflammatory cytokines IL- $1 \beta$, IL- 6 and TNF- $\alpha$. Additionally, the capacity to anatgonize the arachidonic acid cascade was highlighted in previous studies. In particular, the antagonism towards the COX-2 enzyme emerged in the research, and then the ability to antagonize the first phase of the active inflammation by inhibiting prostaglandins. Furthermore, the potential LOX inhibitory capability could propose the EO as a COX-2LOX dual inhibitory natural compound, which might be promising against inflammation and tissue damage.

Among the non-cannabinoid terpenoid fractions, (E)- $\beta$-caryophyllene has been suggested to possess an important role in the anti-inflammatory activity of the oil. Interestingly, the molecule has a strong affinity to CB2, where it inhibits the release of cytokines from LPSstimulated monocytes, such as TNF- $\alpha$ and IL- $1 \beta$ expression. Furthermore, studies in animals have proved the relevance of (E)- $\beta$-caryophyllene in preventing ischemic/reperfusion oxidative injury when the oil was administered as a dietary assumption. In this context, studies strengthened the adjuvant capacity of $\alpha$-pinene merged with caryophyllene, in PIL oils by the high anti-inflammatory properties. Conversely, when merged with the non-cannabinoid myrcene, caryophyllene contributed to inhibit nitric oxide production and IL-1 $\beta$-induced iNOS mRNA, NF-kB and other catabolic and inflammatory mediators of importance in rheumatoid arthritis. Regarding the limonene fraction of the EO, it should be relevant in the oxidative stress-related diseases by inhibiting pro-inflammatory mediators, leukocyte migration and the vascular permeability.

The high antioxidant property of the extracts is remarkable in the literature. This fact has been explained by the richness of polyphenols, including tannins and flavonoids, and sterols showing high protection against free radical damage. Animal testing further supports the in vitro anti-ROS potency of PIL extracts, with a higher effect in comparison to NADPHs. Notably, the aqueous formulations show great capacity in inhibiting lipid peroxidation. The mechanism is interpreted as scavenging of peroxyl radicals, which suggests PIL to be preventive in cardiovascular disease and cancer. Furthermore, the antioxidant properties of polyphenols in PIL extracts should be beneficial in therapy and prevention of gastrointestinal diseases, where oxidative stress has been shown to damage the barrier protection, leading to intestinal pathologies. In this matter, the proved capacity of polyphenols to manage the microbial and metabolomic patterns in the body might represent an additional advantage. These facts should validate scientifically the popular use of PIL extracts to resolve infections and persistent inflammation at the intestinal tract, proposing the materials as natural agents in prevention and therapy of gastro-intestinal diseases.

In regard to the antimicrobial activity, the capacity of the oil and extracts against periodontal bacteria has been largely documented. These evidences can prove scientifically the popular use of PIL in the relief of gingival bleeding and tooth ache. The ability against periodontal bacteria, further ameliorated by the anti-inflammatory potency, is attractive 
with regards to a possible use of the EO to antagonize gingivitis, as a primary strategy to prevent periodontitis and as a secondary preventive strategy to prevent recurrent periodontitis after periodontal surgery. The possibility to formulate PIL derivates as potential oral health care products or therapeutics in periodontal disease is further strengthened by the largely documented biocompatibility and antioxidant capacity.

Other important considerations concern the activity of PIL against Candida infections. PIL inhibits the growth of C. albicans and C. glabrata with low MICs. In addition, the prevention of arachidonic acid oxidation by COX-2 and LOX antagonism by PIL oil should be of interest for inhibiting the development of Candida biofilm and disseminations. Subsequently, PIL could act directly against the yeast and indirectly against its virulence, with no oral cytotoxicity.

Taken together, all the above argumentations propose PIL as a nutraceutical, and also as a therapeutic agent against a wide range of diseases based on inflammation and infections. Further research may include the activity of PIL not only on planktonic microorganisms but also on biofilms. It should verify the best and most efficient preparation method of PIL plant material related to its activity.

Funding: This research received no external funding.

Data Availability Statement: Data is contained within the article.

Conflicts of Interest: The authors declare no conflict of interest.

\section{References}

1. Thabit, A.K.; Crandon, J.L.; Nicolau, D.P. Antimicrobial resistance: Impact on clinical and economic outcomes and the need for new antimicrobials. Expert Opin. Pharmacother. 2015, 16, 159-177. [CrossRef]

2. Fiala, C.; Pasic, M.D. Aspirin: Bitter pill or miracle drug? Clin. Biochem. 2020, 85, 1-4. [CrossRef]

3. Zhu, W.; Wang, Z.; Sun, Y.; Yang, B.; Wang, Q.; Kuang, H. Traditional uses, phytochemistry and pharmacology of genus Syringa: A comprehensive review. J. Ethnopharmacol. 2021, 266, 113465. [CrossRef] [PubMed]

4. El Omari, N.; Ezzahrae Guaouguaou, F.; El Menyiy, N.; Benali, T.; Aanniz, T.; Chamkhi, I.; Balahbib, A.; Taha, D.; Shariati, M.A.; Zengin, G.; et al. Phytochemical and biological activities of Pinus halepensis mill., and their ethnomedicinal use. J. Ethnopharmacol. 2021, 268, 113661. [CrossRef]

5. Newman, D.J.; Cragg, G.M. Natural Products As Sources of New Drugs over the 30 Years from 1981 to 2010. J. Nat. Prod. 2012, 75, 311-335. [CrossRef]

6. De Cássia da Silveira e Sá, R.; Andrade, L.N.; de Sousa, D.P. A review on anti-inflammatory activity of monoterpenes. Molecules 2013, 18, 1227-1254. [CrossRef]

7. Gonçalves, E.C.D.; Baldasso, G.M.; Bicca, M.A.; Paes, R.S.; Capasso, R.; Dutra, R.C. Terpenoids, Cannabimimetic Ligands, beyond the Cannabis Plant. Molecules 2020, 25, 1567. [CrossRef] [PubMed]

8. McNaught, A.D.; Wilkinson, A. IUPAC Compendium of Chemical Terminology, 2nd ed.; Blackwell Science Publications: Oxford, UK, 1997.

9. Russo, E.B. Cannabis Pharmacology: The Usual Suspects and a Few Promising Leads. Adv. Pharmacol. 2017, 80, 67-134. [CrossRef]

10. Kim, T.; Song, B.; Cho, K.S.; Lee, I.-S. Therapeutic Potential of Volatile Terpenes and Terpenoids from Forests for Inflammatory Diseases. Int. J. Mol. Sci. 2020, 21, 2187. [CrossRef]

11. Basu, A.; Masek, E.; Ebersole, J.L. Dietary Polyphenols and Periodontitis-A Mini-Review of Literature. Molecules 2018, 23, 1786. [CrossRef] [PubMed]

12. Quartu, M.; Serra, M.P.; Boi, M.; Pillolla, G.; Melis, T.; Poddighe, L.; Del Fiacco, M.; Falconieri, D.; Carta, G.; Murru, E.; et al. Effect of acute administration of Pistacia lentiscus L. essential oil on rat cerebral cortex following transient bilateral common carotid artery occlusion. Lipids Health Dis. 2012, 11, 8. [CrossRef]

13. Pachi, V.K.; Mikropoulou, E.V.; Gkiouvetidis, P.; Siafakas, K.; Argyropoulou, A.; Angelis, A.; Mitakou, S.; Halabalaki, M. Traditional uses, phytochemistry and pharmacology of Chios mastic gum (Pistacia lentiscus var. Chia, Anacardiaceae): A review. J. Ethnopharmacol. 2020, 254, 112485. [CrossRef]

14. Papada, E.; Kaliora, A.C. Antioxidant and Anti-Inflammatory Properties of Mastiha: A Review of Preclinical and Clinical Studies. Antioxidants 2019, 8, 208. [CrossRef]

15. Piccolella, S.; Nocera, P.; Carillo, P.; Woodrow, P.; Greco, V.; Manti, L.; Fiorentino, A.; Pacifico, S. An Apolar Pistacia Lentiscus L. Leaf Extract: GC-MS Metabolic Profiling and Evaluation of Cytotoxicity and Apoptosis Inducing Effects on SH-SY5Y and SK-N-BE(2)C Cell Lines. Food Chem. Toxicol. 2016, 95, 64-74. [CrossRef] [PubMed]

16. Zohary, M. A Mono-Graphical Study of the Genus Pistacia. Palest. J. Bot. Jerus. Ser. 1952, 5, 187-228.

17. Rauf, A.; Patel, S.; Uddin, G.; Siddiqui, B.S.; Ahmad, B.; Muhammad, N.; Mabkhot, Y.N.; Hadda, T.B. Phytochemical, ethnomedicinal uses and pharmacological profile of genus Pistacia. Biomed. Pharmacother. 2017, 86, 393-404. [CrossRef] 
18. Treitler, J.T.; Drissen, T.; Stadtmann, R.; Zerbe, S.; Mantilla-Contreras, J. Complementing endozoochorous seed dispersal patterns by donkeys and goats in a semi-natural island ecosystem. BMC Ecol. 2017, 17, 42. [CrossRef]

19. Barra, A.; Coroneo, V.; Dessi, S.; Cabras, P.; Angioni, A. Characterization of the volatile constituents in the essential oil of Pistacia lentiscus L. from different origins and its antifungal and antioxidant activity. J. Agric. Food Chem. 2007, 55, 7093-7098. [CrossRef] [PubMed]

20. Rostas, M.; Maag, D.; Ikegami, M.; Inbar, M. Gall volatiles defend aphids against a browsing mammal. BMC Evol. Biol. 2013, 13, 193. [CrossRef]

21. Inbar, M.; Wink, M.; Wool, D. The evolution of host plant manipulation by insects: Molecular and ecological evidence from gall-forming aphids on Pistacia. Mol. Phylogenet. Evol. 2004, 32, 504-511. [CrossRef]

22. Rand, K.; Bar, E.; Ben-Ari, M.; Lewinsohn, E.; Inbar, M. The mono-and sesquiterpene content of aphid-induced galls on Pistacia palaestina is not a simple reflection of their composition in intact leaves. J. Chem. Ecol. 2014, 40, 632-642. [CrossRef]

23. Elgubbi, H.; Alfageih, L.; Zorab, A.; Elmeheshi, F. Pistacia lentiscus tree and its role in riddance of some environmental polluters. EC Nutr. 2017, 10, 8-14.

24. Cabiddu, A.; Delgadillo-Puga, C.; Decandia, M.; Molle, A.G. Extensive ruminant production systems and milk quality with emphasis on unsaturated fatty acids, volatile compounds, antioxidant protection degree and phenol content. Animals 2019,9 , 771. [CrossRef]

25. Ballero, M.; Poli, F.; Sacchetti, G.; Loi, M.C. Ethnobotanical research in the territory of Fluminimaggiore (south-western Sardinia). Fitoterapia 2001, 72, 788-801. [CrossRef]

26. Palmese, M.T.; Uncini Manganelli, R.E.; Tomei, P.E. An ethno-pharmacobotanical survey in the Sarrabus district (south-east Sardinia). Fitoterapia 2001, 72, 619-643. [CrossRef]

27. Leporatti, M.L.; Ghedira, K. Comparative analysis of medicinal plants used in traditional medicine in Italy and Tunisia. J. Ethnobiol. Ethnomed. 2009, 5, 31. [CrossRef]

28. Leonti, M.; Staub, P.O.; Cabras, S.; Castellanos, M.E.; Casu, L. From cumulative cultural transmission to evidence-based medicine: Evolution of medicinal plant knowledge in Southern Italy. Front. Pharmacol. 2015, 6, 207. [CrossRef]

29. Maxia, A.; Lancioni, M.C.; Balia, A.N.; Alborghetti, R.; Pieroni, A.; Loi, M.C. Medical ethnobotany of the Tabarkins, a Northern Italian (Ligurian) minority in south-western Sardinia. Genet. Resour. Crop. Evol. 2008, 55, 911-924. [CrossRef]

30. Di Rosa, A. Erbe e Piante Medicinali in Sardegna, 3rd ed.; Carlo Delfino Ed.: Sassari, Italy, 2018.

31. Loi, M.C.; Frajus, L.; Maxia, A. Le piante utilizzate nella medicina popolare nel territorio Di Gesturi (Sardegna Centro-Meridionale). Atti. Soc. Tosc. Sci. Flat. Mem. 2002, 109, 167-176.

32. Scherrer, A.M.; Motti, R.; Weckerle, C.S. Traditional plant use in the areas of Monte Vesole and Ascea, Cilento National Park (Campania, Southern Italy). J. Ethnopharmacol. 2005, 97, 129-143. [CrossRef]

33. Benitez, G.; Gonzalez-Tejero, M.R.; Molero-Mesa, J. Knowledge of ethnoveterinary medicine in the Province of Granada, Andalusia, Spain. J. Ethnopharmacol. 2012, 139, 429-439. [CrossRef]

34. Gras, A.; Serrasolses, G.; Valles, J.; Garnatje, T. Traditional knowledge in semi-rural close to industrial areas: Ethnobotanical studies in western Girones (Catalonia, Iberian Peninsula). J. Ethnobiol. Ethnomed. 2019, 15, 19. [CrossRef]

35. Trabelsi, H.; Cherif, O.A.; Sakouhi, F.; Villeneuve, P.; Renaud, J.; Barouh, N.; Boukhchina, S.; Mayer, P. Total lipid content, fatty acids and 4-desmethylsterols accumulation in developing fruit of Pistacia lentiscus L. growing wild in Tunisia. Food Chem. 2012, 131, 434-440. [CrossRef]

36. Saiah, H.; Allem, R.; Kebir, F.Z.R. Antioxidant and antibacterial activities of six Algerian medicinal plants. Int. J. Pharm. Pharm. Sci. 2016, 8, 367-374.

37. Jamila, F.; Mostafa, E. Ethnobotanical survey of medicinal plants used by people in Oriental Morocco to manage various ailments. J. Ethnopharmacol. 2014, 154, 76-87. [CrossRef]

38. Janakat, S.; Al-Merie, H. Evaluation of hepatoprotective effect of Pistacia lentiscus, Phillyrea latifolia and Nicotiana glauca. J. Ethnopharmacol. 2002, 83, 135-138. [CrossRef]

39. Lev, E.; Amar, Z. Ethnopharmacological survey of traditional drugs sold in the Kingdom of Jordan. J. Ethnopharmacol. 2002, 82, 131-145. [CrossRef]

40. Lev, E. Ethno-diversity within current ethno-pharmacology as part of Israeli traditional medicine-A review. J. Ethnobiol. Ethnomed. 2006, 2, 4. [CrossRef]

41. Rahimi, R.; Mozaffari, S.; Abdollahi, M. On the use of herbal medicines in management of inflammatory bowel diseases: A systematic review of animal and human studies. Dig. Sci. 2009, 54, 471-480. [CrossRef]

42. Farzaei, M.H.; Shams-Ardekani, M.R.; Abbasabadi, Z.; Rahimi, R. Scientific evaluation of edible fruits and spices used for the treatment of peptic ulcer in traditional Iranian medicine. ISRN Gastroenterol. 2013, 2013, 136932. [CrossRef]

43. Giner-Larza, E.M.; Manez, S.; Recio, M.C.; Giner, R.M.; Prieto, J.M.; Cerda-Nicolas, M.; Rios, J.L. Oleanonic acid, a 3-oxotriterpene from Pistacia, inhibits leukotriene synthesis and has anti-inflammatory activity. Eur. J. Pharmacol. 2001, 428, 137-143. [CrossRef]

44. Missoun, F.; Bouabedelli, F.; Benhamimed, E.; Baghdad, A.; Djebli, N. Phytochemical study and antibacterial activity of different extracts of Pistacia lentiscus L collected from Dahra Region West of Algeria. J. Fundam. Appl. Sci. 2017, 9, 669-684. [CrossRef]

45. Piluzza, G.; Virdis, S.; Serralutzu, F.; Bullitta, S. Uses of plants, animal and mineral substances in Mediterranean ethno-veterinary practices for the care of small ruminants. J. Ethnopharmacol. 2015, 168, 87-99. [CrossRef] 
46. Tassou, C.C.; Chorianopoulos, N.G.; Skandamis, P.N.; Nychas, G.J.E. Herbs, spices and their active components as natural antimicrobials in foods. In Handbook of Herbs and Spices; Peter, K.V., Ed.; Woodhead Publishing: Cambridge, UK, 2012; Volume 2, pp. 17-50.

47. Farmacopea Ufficiale della Repubblica Italiana; Istituto Poligrafico e Zecca dello Stato: Roma, Italy, 2008.

48. Franz, C.; Novak, J. Sources of essential oils. In Handbook of Essential Oils: Science, Technology, and Applications; Baser, K.H.C., Buchbauer, G., Eds.; CPR Press Taylor \& Francis Group: Boca Raton, FL, USA, 2010; pp. 39-82.

49. Milia, E.; Usai, M.; Szotakova, B.; Elstnerova, M.; Kralova, V.; D’Hallewin, G.; Spissu, Y.; Barberis, A.; Marchetti, M.; Bortone, A.; et al. The Pharmaceutical Ability of Pistacia Lentiscus L. Leaves Essential Oil Against Periodontal Bacteria and Candida Sp. and Its Anti-Inflammatory Potential. Antibiotics 2020, 9, 281. [CrossRef]

50. Russo, E.B. Taming THC: Potential Cannabis Synergy and Phytocannabinoid-Terpenoid Entourage Effects. Br. J. Pharmacol. 2011, 163, 1344-1364. [CrossRef]

51. Gardeli, C.; Vassiliki, P.; Athanasios, M.; Kibouris, T.; Komaitis, M. Essential Oil Composition of Pistacia Lentiscus L. and Myrtus Communis L.: Evaluation of Antioxidant Capacity of Methanolic Extracts. Food Chem. 2008, 107, 1120-1130. [CrossRef]

52. De Pooter, H.L.; Schamp, N.M.; Aboutabl, E.A.; Tohamy, S.F.; Doss, S.L. Essential oils from the leaves of three Pistacia species grown in Egypt. Flavour Fragr. J. 1991, 6, 229-232. [CrossRef]

53. Boelens, M.H.; Jimenez, R. Chemical composition of the essential oils from the gum and from various parts of Pistacia lentiscus 1. (mastic gum tree). Flavour Fragr. J. 1991, 6, 271-275. [CrossRef]

54. Zrira, S.; Elamrani, A.; Benjilali, B. Chemical composition of the essential oil of Pistacia lentiscus L. from Morocco-A seasonal variation. Flavour Fragr. J. 2003, 18, 475-480. [CrossRef]

55. Ben Douissa, F.; Hayder, N.; Chekir-Ghedira, L.; Hammami, M.; Ghedira, K.; Mariotte, A.M.; Dijoux-Franca, M.G. New study of the essential oil from leaves of Pistacia lentiscus L. (Anacardiaceae) from Tunisia. Flavour Fragr. J. 2005, 20, 410-414. [CrossRef]

56. Bozorgi, M.; Memariani, Z.; Mobli, M.; Salehi Surmaghi, M.H.; Shams-Ardekani, M.R.; Rahimi, R. Five Pistacia species (P. vera, P. atlantica, P. terebinthus, P. khinjuk, and P. lentiscus): A Review of Their Traditional Uses, Phytochemistry, and Pharmacology. Sci. World J. 2013, 2013, 219815. [CrossRef]

57. Vogelman, T.C. Plant tissue optics. Rev. Plant Physiol. Plant Mol. Biol. 1993, 44, 231-251. [CrossRef]

58. Orru, G.; Demontis, C.; Mameli, A.; Tuveri, E.; Coni, P.; Pichiri, G.; Coghe, F.; Rosa, A.; Rossi, P.; D’Hallewin, G. The Selective Interaction of Pistacia Lentiscus Oil vs. Human Streptococci, an Old Functional Food Revisited with New Tools. Front. Microbiol. 2017, 8, 2067. [CrossRef] [PubMed]

59. Stagos, D. Antioxidant Activity of Polyphenolic Plant Extracts. Antioxidants 2019, 9, 19. [CrossRef] [PubMed]

60. Romani, A.; Pinelli, P.; Galardi, C.; Mulinacci, N.; Tattini, M. Identification and quantification of galloyl derivatives, flavonoid glycosides and anthocyanins in leaves of Pistacia lentiscus L. Phytochem. Anal. 2002, 13, 79-86. [CrossRef] [PubMed]

61. Rodriguez-Perez, C.; Quirantes-Pine, R.; Amessis-Ouchemoukh, N.; Madani, K.; Segura-Carretero, A.; Fernandez-Gutierrez, A. A metabolite-profiling approach allows the identification of new compounds from Pistacia lentiscus leaves. J. Pharm. Biomed. Anal. 2013, 77, 167-174. [CrossRef] [PubMed]

62. Magiatis, P.; Melliou, E.; Skaltsounis, A.L.; Chinou, I.B.; Mitaku, S. Chemical composition and antimicrobial activity of the essential oils of Pistacia lentiscus var. chia. Planta Med. 1999, 65, 749-752. [CrossRef]

63. Duru, M.E.; Cakir, A.; Kordali, S.; Zengin, H.; Harmandar, M.; Izumi, S.; Hirata, T. Chemical composition and antifungal properties of essential oils of three Pistacia species. Fitoterapia 2003, 74, 170-176. [CrossRef]

64. Dob, T.; Dahmane, D.; Chelghoum, C. Chemical composition of the essential oils of Pistacia lentiscus L. from Algeria. J. Essent. Oil Res. 2006, 18, 335-338. [CrossRef]

65. Said, S.A.; Fernandez, C.; Greff, S.; Torre, F.; Derridj, A.; Gauquelin, T.; Mevy, J.P. Inter-Population Variability of Terpenoid Composition in Leaves of Pistacia lentiscus L. from Algeria: A Chemoecological Approach. Molecules 2011, 16, $2646-2657$. [CrossRef]

66. Aouinti, F.; Imelouane, B.; Tahri, M.; Wathelet, J.P.; Amhamdi, H.; Elbachiri, A. New study of the essential oil, mineral composition and antibacterial activity of Pistacia lentiscus L. from Eastern Morocco. Res. Chem. Intermed. 2014, 40, 2873-2886. [CrossRef]

67. Bampouli, A.; Kyriakopoulou, K.; Papaefstathiou, G.; Louli, V.; Krokida, M.; Magoulas, K. Comparison of different extraction methods of Pistacia lentiscus var. chia leaves: Yield, antioxidant activity and essential oil chemical composition. J. Appl. Res. Med. Aromat. Plants 2014, 1, 81-91. [CrossRef]

68. Mezni, F.; Aouadhi, C.; Khouja, M.L.; Khaldi, A.; Maaroufi, A. In vitro antimicrobial activity of Pistacia lentiscus L. edible oil and phenolic extract. Nat. Prod. Res. 2015, 29, 565-570. [CrossRef] [PubMed]

69. Aissi, O.; Boussaid, M.; Messaoud, C. Essential oil composition in natural populations of Pistacia lentiscus L. from Tunisia: Effect of ecological factors and incidence on antioxidant and antiacetylcholinesterase activities. Ind. Crop. Prod. 2016, 91, 56-65. [CrossRef]

70. Ben Khedir, S.; Mzid, M.; Bardaa, S.; Moalla, D.; Sahnoun, Z.; Rebai, T. In vivo evaluation of the anti-inflammatory effect of Pistacia lentiscus fruit oil and its effects on oxidative stress. Evid. Based Complement. Altern. Med. 2016, 2016, 6108203. [CrossRef]

71. Buriani, A.; Fortinguerra, S.; Sorrenti, V.; Dall'Acqua, S.; Innocenti, G.; Montopoli, M.; Gabbia, D.; Carrara, M. Human Adenocarcinoma Cell Line Sensitivity to Essential Oil Phytocomplexes from Pistacia Species: A Multivariate Approach. Molecules 2017, 22, 1336. [CrossRef] 
72. Marengo, A.; Piras, A.; Falconieri, D.; Porcedda, S.; Caboni, P.; Cortis, P.; Foddis, C.; Loi, C.; Goncalves, M.J.; Salgueiro, L.; et al. Chemical and biomolecular analyses to discriminate three taxa of Pistacia genus from Sardinia Island (Italy) and their antifungal activity. Nat. Prod. Res. 2018, 32, 2766-2774. [CrossRef] [PubMed]

73. Ammari, M.; Othman, H.; Hajri, A.; Sakly, M.; Abdelmelek, H. Pistacia lentiscus oil attenuates memory dysfunction and decreases levels of biomarkers of oxidative stress induced by lipopolysaccharide in rats. Brain Res. Bull. 2018, 140, 140-147. [CrossRef]

74. Yosr, Z.; Imen, B.H.Y.; Rym, J.; Chokri, M.; Mohamed, B. Sex-related differences in essential oil composition, phenol contents and antioxidant activity of aerial parts in Pistacia lentiscus L. during seasons. Ind. Crop. Prod. 2018, 121, 151-159. [CrossRef]

75. Bouyahya, A.; Assemian, I.C.C.; Mouzount, H.; Bourais, I.; Et-Touys, A.; Fellah, H.; Benjouad, A.; Dakka, N.; Bakri, Y. Could volatile compounds from leaves and fruits of Pistacia lentiscus constitute a novel source of anticancer, antioxidant, antiparasitic and antibacterial drugs? Ind. Crop. Prod. 2019, 128, 62-69. [CrossRef]

76. Longo, L.; Scardino, A.; Vasapollo, G. Identification and quantification of anthocyanins in the berries of Pistacia lentiscus L., Phillyrea latifolia L. and Rubia peregrina L. Innov. Food Sci. Emerg. Technol. 2007, 8, 360-364. [CrossRef]

77. Pacifico, S.; Piccolella, S.; Marciano, S.; Galasso, S.; Nocera, P.; Piscopo, V.; Fiorentino, A.; Monaco, P. LC-MS/MS Profiling of a Mastic Leaf Phenol Enriched Extract and Its Effects on H2O2 and A beta(25-35) Oxidative Injury in SK-B-NE(C)-2 Cells. J. Agric. Food Chem. 2014, 62, 11957-11966. [CrossRef] [PubMed]

78. Mezni, F.; Slama, A.; Ksouri, R.; Hamdaoui, G.; Khouja, M.L.; Khaldi, A. Phenolic profile and effect of growing area on Pistacia lentiscus seed oil. Food Chem. 2018, 257, 206-210. [CrossRef]

79. Gori, A.; Nascimento, L.B.; Ferrini, F.; Centritto, M.; Brunetti, C. Seasonal and Diurnal Variation in Leaf Phenolics of Three Medicinal Mediterranean Wild Species: What Is the Best Harvesting Moment to Obtain the Richest and the Most Antioxidant Extracts? Molecules 2020, 25, 956. [CrossRef]

80. El Bishbishy, M.H.; Gad, H.A.; Aborehab, N.M. Chemometric discrimination of three Pistacia species via their metabolic profiling and their possible in vitro effects on memory functions. J. Pharm. Biomed. Anal. 2020, 177, 112840. [CrossRef]

81. Zeng, Y.; Song, J.-X.; Shen, X.-C. Herbal remedies supply a novel prospect for the treatment of atherosclerosis: A review of current mechanism studies. Phytother. Res. PTR 2012, 26, 159-167. [CrossRef] [PubMed]

82. Jiang, F.; Chen, W.; Yi, K.J.; Wu, Z.Q.; Si, Y.L.; Han, W.D.; Zhao, Y.L. The evaluation of catechins that contain a galloyl moiety as potential HIV-1 integrase inhibitors. Clin. Immunol. 2010, 137, 347-356. [CrossRef]

83. Wang, B.; Yao, M.; Lv, L.; Ling, Z.; Li, L. The Human Microbiota in Health and Disease. Engineering 2017, 3, 71-82. [CrossRef]

84. Zhao, Y.L.; Jiang, F.; Liu, P.; Chen, W.; Yi, K.J. Catechins containing a galloyl moiety as potential anti-HIV-1 compounds. Drug Discov. Today 2012, 17, 630-635. [CrossRef]

85. Campbell, E.L.; Colgan, S.P. Control and dysregulation of redox signalling in the gastrointestinal tract. Nat. Rev. Gastroenterol. Hepatol. 2019, 16, 106-120. [CrossRef]

86. Benhammou, N.; Bekkara, F.A.; Panovska, T.K. Antioxidant and antimicrobial activities of the Pistacia lentiscus and Pistacia atlantica extracts. Afr. J. Pharm. Pharmacol. 2008, 2, 22-28.

87. Vamanu, E.; Gatea, F. Correlations between Microbiota Bioactivity and Bioavailability of Functional Compounds: A Mini-Review. Biomedicines 2020, 8, 39. [CrossRef] [PubMed]

88. Zhou, Y.; Zheng, J.; Li, Y.; Xu, D.P.; Li, S.; Chen, Y.M.; Li, H.B. Natural Polyphenols for Prevention and Treatment of Cancer. Nutrients 2016, 8, 515. [CrossRef] [PubMed]

89. González, R.; Ballester, I.; López-Posadas, R.; Suárez, M.D.; Zarzuelo, A.; Martínez-Augustin, O.; Sánchez de Medina, F. Effects of flavonoids and other polyphenols on inflammation. Crit. Rev. Food Sci. Nutr. 2011, 51, 331-362. [CrossRef] [PubMed]

90. Kim, H.P.; Son, K.H.; Chang, H.W.; Kang, S.S. Anti-inflammatory plant flavonoids and cellular action mechanisms. J. Pharmacol. Sci. 2004, 96, 229-245. [CrossRef]

91. Comalada, M.; Ballester, I.; Bailón, E.; Sierra, S.; Xaus, J.; Gálvez, J.; de Medina, F.S.; Zarzuelo, A. Inhibition of pro-inflammatory markers in primary bone marrow-derived mouse macrophages by naturally occurring flavonoids: Analysis of the structureactivity relationship. Biochem. Pharmacol. 2006, 72, 1010-1021. [CrossRef] [PubMed]

92. Malireddy, S.; Kotha, S.R.; Secor, J.D.; Gurney, T.O.; Abbott, J.L.; Maulik, G.; Maddipati, K.R.; Parinandi, N.L. Phytochemical Antioxidants Modulate Mammalian Cellular Epigenome: Implications in Health and Disease. Antioxid. Redox Signal. 2012, 17, 327-339. [CrossRef]

93. Santangelo, C.; Varì, R.; Scazzocchio, B.; Di Benedetto, R.; Filesi, C.; Masella, R. Polyphenols, intracellular signalling and inflammation. Ann. Ist. Super. Sanita 2007, 43, 394-405.

94. Atmani, D.; Chaher, N.; Berboucha, M.; Ayouni, K.; Lounis, H.; Boudaoud, H.; Debbache, N.; Atmani, D. Antioxidant capacity and phenol content of selected Algerian medicinal plants. Food Chem. 2009, 112, 303-309. [CrossRef]

95. Bullitta, S.; Piluzza, G.; Manunta, M.D.I. Cell-based and chemical assays of the ability to modulate the production of intracellular Reactive Oxygen Species of eleven Mediterranean plant species related to ethnobotanic traditions. Genet. Resour. Crop. Evol. 2013, 60, 403-412. [CrossRef]

96. Remila, S.; Atmani-Kilani, D.; Delemasure, S.; Connat, J.L.; Azib, L.; Richard, T.; Atmani, D. Antioxidant, cytoprotective, antiinflammatory and anticancer activities of Pistacia lentiscus (Anacardiaceae) leaf and fruit extracts. Eur. J. Integr. Med. 2015, 7, 274-286. [CrossRef]

97. Dellai, A.; Souissi, H.; Borgi, W.; Bouraoui, A.; Chouchane, N. Antiinflammatory and antiulcerogenic activities of Pistacia lentiscus L. leaves extracts. Ind. Crop. Prod. 2013, 49, 879-882. [CrossRef] 
98. Schieber, M.; Chandel, N.S. ROS function in redox signaling and oxidative stress. Curr. Biol. 2014, 24, R453-R462. [CrossRef]

99. García-Martínez, O.; De Luna-Bertos, E.; Ramos-Torrecillas, J.; Ruiz, C.; Milia, E.; Lorenzo, M.L.; Jimenez, B.; Sánchez-Ortiz, A.; Rivas, A. Phenolic Compounds in Extra Virgin Olive Oil Stimulate Human Osteoblastic Cell Proliferation. PLoS ONE 2016, 11, e0150045. [CrossRef] [PubMed]

100. Chirumbolo, S.; Bjørklund, G. Sulforaphane and 5-fluorouracil synergistically inducing autophagy in breast cancer: A possible role for the Nrf2-Keap1-ARE signaling? Food Chem. Toxicol. 2018, 112, 414-415. [CrossRef] [PubMed]

101. Zhou, Y.; Jiang, Z.; Lu, H.; Xu, Z.; Tong, R.; Shi, J.; Jia, G. Recent Advances of Natural Polyphenols Activators for Keap1-Nrf2 Signaling Pathway. Chem. Biodivers. 2019, 16, e1900400. [CrossRef]

102. Baratto, M.C.; Tattini, M.; Galardi, C.; Pinelli, P.; Romani, A.; Visioli, F.; Basosi, R.; Pogni, R. Antioxidant activity of galloyl quinic derivatives isolated from P-lentiscus leaves. Free. Radic. Res. 2003, 37, 405-412. [CrossRef]

103. Catalani, S.; Palma, F.; Battistelli, S.; Benedetti, S. Oxidative stress and apoptosis induction in human thyroid carcinoma cells exposed to the essential oil from Pistacia lentiscus aerial parts. PLoS ONE 2017, 12, e0172138. [CrossRef]

104. Cappadone, C.; Mandrone, M.; Chiocchio, I.; Sanna, C.; Malucelli, E.; Bassi, V.; Picone, G.; Poli, F. Antitumor Potential and Phytochemical Profile of Plants from Sardinia (Italy), a Hotspot for Biodiversity in the Mediterranean Basin. Plants 2020, 9, 26. [CrossRef] [PubMed]

105. Janssen-Heininger, Y.M.W.; Poynter, M.E.; Baeuerle, P.A. Recent advances towards understanding redox mechanisms in the activation of nuclear factor kappa B. Free Radic. Biol. Med. 2000, 28, 1317-1327. [CrossRef]

106. Quartu, M.; Poddighe, L.; Melis, T.; Serra, M.P.; Boi, M.; Lisai, S.; Carta, G.; Murru, E.; Muredda, L.; Collu, M.; et al. Involvement of the endocannabinoid system in the physiological response to transient common carotid artery occlusion and reperfusion. Lipids Health Dis. 2017, 16, 14. [CrossRef] [PubMed]

107. Iauk, L.; Ragusa, S.; Rapisarda, A.; Franco, S.; Nicolosi, V.M. In vitro antimicrobial activity of Pistacia lentiscus L. extracts: Preliminary report. J. Chemother. 1996, 8, 207-209. [CrossRef]

108. Mandrone, M.; Bonvicini, F.; Lianza, M.; Sanna, C.; Maxia, A.; Gentilomi, G.A.; Poli, F. Sardinian plants with antimicrobial potential. Biological screening with multivariate data treatment of thirty-six extracts. Ind. Crop. Prod. 2019, 137, 557-565. [CrossRef]

109. Turchetti, B.; Pinelli, P.; Buzzini, P.; Romani, A.; Heimler, D.; Franconi, F.; Martini, A. In vitro antimycotic activity of some plant extracts towards yeast and yeast-like strains. Phytother. Res. 2005, 19, 44-49. [CrossRef] [PubMed]

110. Krishna, G.; Gopalakrishnan, G. Chapter 27-Alternative in Vitro Models for Safety and Toxicity Evaluation of Nutraceuticals. In Nutraceuticals; Gupta, R.C., Ed.; Academic Press: Boston, MA, USA, 2016; pp. 355-385. ISBN 978-0-12-802147-7.

111. Yang, C.S.; Landau, J.M.; Huang, M.T.; Newmark, H.L. Inhibition of Carcinogenesis by Dietary Polyphenolic Compounds. Ann. Rev. Nutr. 2001, 21, 381-406. [CrossRef]

112. Carrol, D.H.; Chassagne, F.; Dettweiler, M.; Quave, C.L. Antibacterial Activity of Plant Species Used for Oral Health against Porphyromonas Gingivalis. PLoS ONE 2020, 15, e0239316. [CrossRef] [PubMed]

113. Tariq, S.; Wani, S.; Rasool, W.; Shafi, K.; Bhat, M.A.; Prabhakar, A.; Shalla, A.H.; Rather, M.A. A comprehensive review of the antibacterial, antifungal and antiviral potential of essential oils and their chemical constituents against drug-resistant microbial pathogens. Microb. Pathog. 2019, 134, 103580. [CrossRef]

114. Djenane, D.; Yanguela, J.; Montanes, L.; Djerbal, M.; Roncales, P. Antimicrobial activity of Pistacia lentiscus and Satureja montana essential oils against Listeria monocytogenes CECT 935 using laboratory media: Efficacy and synergistic potential in minced beef. Food Control. 2011, 22, 1046-1053. [CrossRef]

115. Liu, X.N.; Wang, D.; Yu, C.X.; Li, T.; Liu, J.Q.; Sun, S.J. Potential Antifungal Targets against a Candida Biofilm Based on an Enzyme in the Arachidonic Acid Cascade-A Review. Front. Microbiol. 2016, 7, 1925. [CrossRef] [PubMed] 